\title{
Emploi des vasoconstricteurs en odonto-stomatologie Recommandations
}

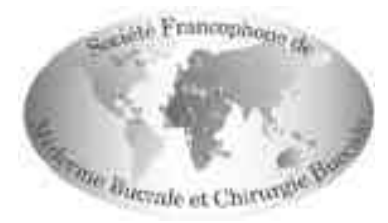

\section{SOCIÉTÉ FRANCOPHONE DE MÉDECINE BUCCALE ET DE CHIRURGIE BUCCALE}

\section{Groupe de travail}

Carlos Madrid (Odontologiste, Toulouse), Rapporteur Bruno Courtois (Odontologiste, Toulouse)

Marc Vironneau (Anesthésiste Réanimateur, Toulouse)

\section{Groupe de lecture}

J acques Bayssière (Odontologiste, Montpellier)

J ean Pierre Bernard (Stomatologiste, Genève)

J ean Loup Coudert (Odontologiste, Lyon)

Damien Duran (Odontologiste, Toulouse)

Ahmed Feki (Odontologiste, Strasbourg)

J ean Christophe Fricain (Odontologiste, Bordeaux)

Patrick G irard (Odontologiste, Paris)

J ean Claude Harnet (Odontologiste, Strasbourg)

J acques J eandot (Stomatologiste, Bordeaux)

Benoît Lefèvre (Odontologiste, Reims)

Dan Longrois (Anesthésiste-Réanimateur, Nancy)

Louis Maman (Odontologiste, Paris)

Gilbert de Mello (Odontologiste, Rennes)

Guillaume Penel (Odontologiste, Lille)

François Prédine-Hug (Odontologiste, Brest)

Yvon Roche (Odontologiste, Paris)

J acky Samson (Stomatologiste, Genève)

J acques Henri Torres (Stomatologiste, Montpellier)

Daniel Viennet (Odontologiste, Nancy)

\section{MÉTHODOLOGIE GÉNÉRALE}

Ces recommandations sur l'emploi des vasoconstricteurs en odonto-stomatologie ont été élaborées par un groupe de travail au terme d'une analyse de la littérature scientifique et du recueil de l'avis des membres de la Société Francophone de Médecine Buccale et de Chirurgie Buccale en séance scientifique à Metz le 23 mai 2002. Le texte a ensuite été soumis à un groupe de lecture avant d'être définitivement adopté.

Les membres des groupes de travail et de lecture ont été désignés par la Société Francophone de Médecine Buccale et de Chirurgie Buccale. Le groupe de travail était animé par un rapporteur qui a rédigé le document final avant de le proposer et de le discuter avec le groupe de travail puis de le soumettre au comité de lecture.

Une recherche bibliographique systématique a été réalisée par interrogation de la banque de donnée Medline.

Cette bibliographie obtenue par voie automatisée a été complétée par une recherche manuelle. Les membres du groupe de travail et du groupe de lecture ont transmis des articles. Les listes de références citées dans les articles déjà identifiés ont été consultées. médecine buccale chirurgie buccale VOL. $9, \mathrm{~N}^{\circ}$ 2003 page 65 
médecine buccale chirurgie buccale

)L. $9, \mathrm{~N}^{\circ} 2$

2003

page 66
Le rapporteur a utilisé avec le groupe de travail des grilles de lecture destinées à apprécier la qualité méthodologique et le niveau de preuve scientifique de ces documents. Les documents ont été classés selon ces grilles en différentes catégories. Sur la base de cette analyse de la littérature, le groupe de travail a proposé chaque fois que possible des recommandations. Celles-ci ont été basées soit sur un niveau de preuve scientifique, soit, en l'absence de preuve, sur un accord professionnel fort recueilli lors de la séance scientifique de la Société Francophone de Médecine Buccale et de Chirurgie Buccale du 23 mai 2002.

La bibliographie obtenue a été presque totalement utilisée de sorte que le groupe de travail n'a pas jugé utile de séparer bibliographie sélective et bibliographie complémentaire. En revanche la totalité de la bibliographie obtenue a été classée analytiquement selon le niveau de preuve $(\mathrm{NdP})$ à partir de la classification suivante (US Agency for Health Care Policy and Research):

\begin{tabular}{|c|c|}
\hline Niveau I a : & $\begin{array}{l}\text { Preuve de méta-analyse d'essais } \\
\text { randomisés contrôlés (Randomized } \\
\text { Controlled Trials, RCT) }\end{array}$ \\
\hline Niveau I b & Preuve à partir d'au moins un $\mathrm{RCT}$ \\
\hline Niveau II a & $\begin{array}{l}\text { Preuve à partir d'une étude contrôlée } \\
\text { non randomisée }\end{array}$ \\
\hline Niveau II b : & $\begin{array}{l}\text { : Preuve à partir d'une autre étude } \\
\text { expérimentale bien définie }\end{array}$ \\
\hline Niveau III & $\begin{array}{l}\text { Preuve d'étude expérimentale } \\
\text { descriptive bien définie (ceci inclut } \\
\text { les études comparatives, les études } \\
\text { de cohorte, et les études de contrôle } \\
\text { de cas) }\end{array}$ \\
\hline Niveаu IV & erts ou expérience cliniq \\
\hline
\end{tabular}

Les grades $A$, B et $C$ sont attribués aux recommandations en fonction du niveau de preuve de la bibliographique respective :

Grade A : Basée sur un niveau de preuve I

Grade B : Basée sur niveau de preuve II ou III

Grade C : Basée sur un niveau de preuve IV

\section{Stratégie de la recherche documentaire}

La recherche automatisée a été basée sur les mots-clés suivants :

- local anaesthesia

- general anaesthesia

- vasoconstrictors

- nor-epinephrine

- epinephrine

- levonordefrin

- corbadrine

Les mots-clés précédents ont été croisé à :

- dentistry

- maxillofacial surgery

- side effects

- adverse effects

- special patients

\section{Questions retenues}

- Quelle est la place des vasoconstricteurs en odonto-stomatologie?

- Les vasoconstricteurs sont-ils nécessaires en anesthésie odonto-stomatologique?

- Les vasoconstricteurs peuvent-ils être utiles en pratique odonto-stomatologique en dehors de leur association à la substance anesthésique locale?

- Les vasoconstricteurs peuvent-ils être associés aux anesthésiques généraux lors d'une anesthésie générale en odonto-stomatologie?

- Comment choisir la molécule vasoconstrictrice en odonto-stomatologie?

- Quels sont les avantages et désavantages comparés de l'adrénaline et de la noradrénaline?

- Quel est l'intérêt des autres substances ?

- Quelles sont les indications des vasoconstricteurs en odonto-stomatologie?

- Quel doit être le dosage des vasoconstricteurs en anesthésie odonto-stomatologique?

- Quelles sont les interactions médicamenteuses des vasoconstricteurs utilisés en anesthésie odonto-stomatologique?

- Quelles sont les affections qui contre-indiquent les vasoconstric teurs en odonto-stomatologie?

- Les états physiologiques contre-indiquentils les vasoconstricteurs en anesthésie odonto-stomatologique? 


\section{RECOMMANDATIONS}

\section{Technique d'Injection}

1. L'injection d'une solution anesthésique avec ou sans vasoconstricteur doit toujours être effectuée lentement ( $1 \mathrm{ml}$ par minute) et de façon fractionnée afin de surveiller les signes éventuels d'un effet délétère de l'injection. Lorsque l'injection a lieu dans un territoire bien vascularisé, un test d'aspiration négatif est un préalable constant à l'injection de la solution anesthésique avec ou sans vasoconstricteur. La plus petite dose efficace est toujours recommandée. [Grade C]

\section{Indications}

2. L'association d'un vasoconstricteur à la solution anesthésique en anesthésie odontostomatologique locale par infiltration est indiquée car le vasoconstricteur diminue le passage intra-vasculaire du mélange injecté et assure ainsi une augmentation de la durée et de la profondeur de l'anesthésie tout en réduisant les effets systémiques de la solution. [Grade A]

3. II ne paraît pas possible de conclure formellement quant à l'innocuité des cordonnets rétracteurs imbibés de vasoconstricteur employés en prothèse dentaire. Les évaluations chez l'animal semblent montrer que les variations hémodynamiques sont inconstantes. La littérature rapporte un accident grave chez I'homme. [GradeB]

4. Les techniques d'hémostase locale par emploi de vasoconstricteurs purs ou bien mélangés à des substances anesthésiques ou astringentes n'ont fait l'objet d'aucune évaluation publiée présentant un niveau de preuve satisfaisant. Elles sont donc du domaine empirique. Bien que largement diffusées, elles n'ont pas donné lieu à la publication d'accident ou d'incident en rapport avec les vasoconstricteurs. [Grade $\mathbf{C}$ ]

5. L'utilisation d'une solution anesthésique additionnée d'un vasoconstricteur comme moyen de diminuer le saignement et d'abaisser le seuil de l'analgésie chez des patients opérés en chirurgie buccale sous anesthésie générale contribue à diminuer la réponse sympathique à l'agression chirurgicale et à diminuer la profondeur de l'anesthésie générale nécessaire. [Grade B]

\section{Choix de la molécule}

6. L'adrénaline est industriellement et médicalement le chef de file des vasoconstricteurs utilisés seuls ou en association avec un anesthésique local en odonto-stomatologie. II dispose de la plus large casuistique qui confirme une grande sécurité de cette molécule. Les dérivés non-catéchols n'ont pas fait la preuve de leur supériorité à ce jour même chez des patients susceptibles de mal tolérer les catécholamines. [Grade B]

\section{Indications en fonction de la tech- nique anesthésique}

7. L'utilisation d'un vasoconstricteur dans les techniques d'anesthésie ponctuelle (anesthésie intrapulpaire, intraligamentaire et intraseptale) n'est pas indispensable mais améliore considérablement le taux de succès, la durée et la profondeur de l'anesthésie obtenue. Si l'injection est effectuée dans des conditions adéquates, les lésions locales directement imputables au vasoconstricteur sont négligeables et réversibles. Les effets systémiques de ces injections existent mais sont le plus souvent très inférieurs à ceux observés dans les anesthésies par infiltration. [Grade A]

8. L'utilisation d'un vasoconstricteur dans les techniques d'anesthésie locale (para-apicale, anesthésie du nerf lingual, anesthésie du nerf buccal) n'est pas indispensable, mais améliore sensiblement le taux de succès, la durée et la profondeur de l'anesthésie obtenue. [Grade C]

9. L'addition d'un vasoconstricteur à la solution anesthésique n'est pas indispensable pour l'anesthésie au foramen mandibulaire du nerf alvéolaire inférieur. L'addition d'adrénaline aug- médecine buccale chirurgie buccale VOL. $9, \mathrm{~N}^{\circ}$ 2003 page 67 
médecine buccale chirurgie buccale

)L. $9, \mathrm{~N}^{\circ} 2$ 2003 page 68 mente la durée de l'anesthésie mais ne semble pas avoir d'effet décisif sur l'incidence des échecs. Les résultats concernant le taux de succès de l'anesthésie sont contradictoires. Compte tenu de la relation qui existe entre le taux de succès et le volume de solution injectée, l'addition d'un vasoconstricteur pourrait être considérée dans la prévention des effets systémiques des anesthésiques locaux. [GradeC]

\section{Dosage du vasoconstricteur}

10. Les résultats sont contradictoires au sujet du dosage idéal de l'adrénaline dans les solutions à $2 \%$ de lidocaïne. La solution à $1 / 200000$ donne une durée d'action suffisante pour la majorité des actes odonto-stomatologiques. Pour l'articaine à $4 \%$ et la mépivacaïne à $2 \%$, les solutions au $1 / 200000$ devraient être préférées en l'absence de différence significative des performances avec la solution au $1 / 100000$ et parce qu'elles seraient probablement mieux tolérées. [Grade A]

\section{Interactions médicamenteuses}

11. L'attitude face aux patients sous antidépresseurs tricycliques doit être d'écarter la noradrénaline en association aux anesthésiques locaux et d'injecter des doses réduites d'anesthésiques locaux associés à de l'adrénaline au 1/200 000. En pratique la dose injectée devrait être le tiers de la dose totale chez le sujet normal. [GradeC]

12. Les patients sous béta-bloquants cardiosélectifs peuvent recevoir des anesthésies locales avec vasoconstricteur (adrénaline au $1 / 200000$ ). Chez les patients recevant des bétabloquants non sélectifs, il est recommandé d'utiliser des solutions anesthésiques les plus faiblement dosées en vasoconstricteur. [GradeC]

13. Les anesthésiques généraux volatils halogénés ne doivent pas être utilisés avec l'adrénaline. La littérature incite à la prudence quant à l'utilisation d'anesthésiques locaux adrénalinés en cas d'association de thiopental + halothane lors d'une anesthésie générale. [Grade $\mathbf{C}$ ]
14. Les vasoconstricteurs seront proscrits au moins 24 heures après la consommation de cocaïne de la cocaïne pour permettre l'élimination de la drogue et de ses métabolites actifs. [Grade C]

15. Aucun accident n'a été rapporté quant à l'administration d'anesthésique local adrénaliné chez des patients sous antipsychotiques ou alpha-bloquants. Le risque d'interaction entre ces substances est théorique aux doses usuelles en anesthésie odonto-stomatologique.

[Grade C]

16. Il n'existe pas de contre-indication à l'administration d'anesthésique local adrénaliné à des patients sous IMAO sélectifs. [Grade $\mathbf{C}$ ]

\section{Pathologies contre-indiquant les vasoconstricteurs associés à l'anes- thésique local}

17. Le phéochromocytome constitue une contre-indication absolue des vasoconstricteurs. Les malades atteints de cette affection doivent être pris en charge en milieu hospitalier disposant d'une structure de réanimation lorsqu'une anesthésie locale avec ou sans vasoconstricteur est nécessaire. [GradeC]

18. Il paraît souhaitable d'éviter l'association de vasoconstricteurs à l'anesthésique local lors des soins conservateurs et surtout non conservateurs sur un os irradié au delà de $40 \mathrm{~Gy}$. [Grade A]

19. Les injections intra osseuses d'anesthésique local adrénaliné doivent être évitées chez les patients arythmiques. [Grade A]

\section{Pathologies ne contre-indiquant pas les vasoconstricteurs associés à l'anesthésique local}

20. Les patients hyper et hypothyroïdiens stabilisés n'ont pas de troubles majeurs lorsqu'ils sont soumis à un traitement correcteur et mis en présence de catécholamines. Bien 
que le risque théorique de potentialisation thyroxine-adrénaline soit sérieux, il n'existe pas de cas clinique rapporté. [Grade $\mathbf{C}$ ]

21. Les vasoconstricteurs associés à une solution anesthésique ne sont pas contre-indiqués chez un sujet hypertendu stabilisé par le traitement antihypertenseur. [Grade A]

22. En cas d'instabilité tensionnelle associée à d'autres éléments grevant le pronostic, les soins impliquant une anesthésie locale avec vasoconstricteur devront être menés en milieu hospitalier disposant d'une structure de réanimation et effectués sous monitorage. [Grade $\mathbf{C}$ ]

23. Dans les fibrillations auriculaires équilibrées par un traitement adapté le contrôle du stress et de la fréquence cardiaque thérapeutique est essentiel et l'utilisation d'anesthésiques locaux avec vasoconstricteur est indiquée. [Grade C]

24. Les patients sous digoxine et ceux atteints d'arythmies atrio-ventriculaires doivent être traités sous monitorage en milieu hospitalier disposant d'une structure de réanimation lorsqu'une une anesthésie locale avec ou sans vasoconstricteur est nécessaire. [Grade $\mathbf{C}$ ]

25. Les vasoconstricteurs associés à une solution anesthésique ne sont pas contre-indiqués dans les cardiopathies coronariennes stabilisées. [Grade $\mathbf{C}$ ]

26. Les vasoconstricteurs associés à une solution anesthésique ne sont pas contre-indiqués chez les sujets asthmatiques dans le dessein de maîtriser la douleur et d'éviter le stress qui est probablement la principale source de passage à la crise d'asthme au cabinet dentaire. En cas d'asthme cortico-dépendant, le recours à un anesthésique sans vasoconstricteur et donc sans bisulfite est indiqué. [Grade $\mathbf{C}$ ]
27. Les vasoconstricteurs associés à une solution anesthésique ne sont pas contre-indiqués chez les patients ayant présenté une atteinte hépatique virale ou toxique ancienne et guérie. En cas d'atteinte sévère évolutive, l'évaluation de la fonction hépatique est importante. La quantité totale injectée peut devoir être réduite et les intervalles entre les injections augmentés, sans préjudice de l'utilisation d'un vasoconstricteur associé. [Grade $\mathbf{C}$ ]

28. Les vasoconstricteurs associés à une solution anesthésique ne sont pas contre-indiqués chez les patients diabétiques équilibrés de type I ou II. En cas de diabète déséquilibré et instable, avec passage brutal de l'hypo à l'hyperglycémie, les quantités d'anesthésique local avec vasoconstricteur seront modérées de façon à tenir compte du caractère hyperglycémiant de l'adrénaline. [Grade $\mathbf{C}$ ]

\section{États physiologiques et vasocons- tricteurs}

29. Les vasoconstricteurs associés à une solution anesthésique ne sont pas contre-indiqués au cours de la grossesse et de l'allaitement. Les doses usuelles peuvent être utilisées. [Grade C]

30. Les vasoconstricteurs associés à une solution anesthésique ne sont pas contre-indiqués chez l'enfant au delà de six mois. La dose totale d'anesthésique local avec ou sans vasoconstricteur usuelle chez l'adulte sain doit être divisée par 3 en dessous de $15 \mathrm{~kg}$ et par 2 entre 15 et $40 \mathrm{~kg}$. [Grade C]

31. Les vasoconstricteurs associés à une solution d'anesthésique local ne sont pas contreindiqués chez la personne âgée. La dose totale d'anesthésique avec ou sans vasoconstricteur doit être adaptée à l'état métabolique du sujet considéré. [Grade $\mathbf{C}$ ] médecine buccale chirurgie buccale VOL. $9, \mathrm{~N}^{\circ}$ 2003 page 69 
médecine buccale chirurgie buccale

\section{ARGUMENTAIRE}

\section{Place des vasoconstricteurs en odonto-stomatologie}

Les vasoconstricteurs adrénergiques sont parmi les substances thérapeutiques les plus administrées en odonto-stomatologie. Leur utilisation en association avec une substance anesthésique a commencé en 1904 année où Heinreich Braun (1903) spécialiste allemand de renom en anesthésie locale mit au point un mélange adrénalineprocaïne bientôt commercialisé par Hoechst sous la marque Novocaiine ${ }^{\circledR}$ qui devait dominer le marché pendant 50 ans (Yagiela, 1995 [NdP IV]). Les vasoconstricteurs sont également responsables de plus d'interactions médicamenteuses que la plupart des autres substances médicamenteuses spécifiques à l'odonto-stomatologiste (Yagiela, 1999 [NdP IV]). L'adrénaline et les autres dérivés amines sympathicomimétiques sont injectés de façon routinière en association avec des anesthésiques locaux ( $A L)$ pour le contrôle de la douleur, ou bien utilisés seuls sur des cordonnets de rétraction gingivale et dans des solutions injectables ou topiques destinées au contrôle des saignements locaux.

\subsection{Les vasoconstricteurs sont-ils néces- saires en anesthésie stomatologique ?}

Les avantages des vasoconstricteurs en anesthésie odonto-stomatologique sont reconnus de façon universelle par la communauté scientifique et les principaux manuels d'anesthésie locale odonto-stomatologique [NdP IV] y font référence comme à une évidence scientifique (Davies et Lefkowitz, 1981; Malamed, 1997; Berini et Gay, 1997; Gaudy et Arreto, 1999).

On admet généralement que, quelle que soit la voie d'injection, la biodisponiblité des AL de type amino-amide est totale. Après injection une partie de la dose rejoint sa cible tandis qu'une autre fraction passe dans la circulation systémique. Le passage à travers la barrière endothéliale vasculaire est aisé compte tenu de la bonne liposolubilité des AL. Une densité capillaire importante, un débit sanguin local et un coefficient de partage sang/tissus élevés sont autant de facteurs d'accroissement de la résorption systémique qui se trouvent réunis dans la plupart des territoires cibles d'anesthésie locale de la cavité buccale. Diverses interventions pharmacologiques et en particulier l'addition de vasoconstricteurs à la solution AL peuvent modifier sensiblement la résorption systémique bien que celle-ci varie également avec l'effet propre de l'AL utilisé sur la vascularisation locale (Viel et coll, 1997 [NdP IV]; Ackermann et coll, 1988 [NdP III]; Myers et Heckmann,1989 [NdP IIb]).

Le vasoconstricteur agit donc d'abord comme une substance susceptible de ralentir la vitesse d'absorption de la solution anesthésique au point d'injection (Fink et coll, 1978 [NdP Ilb]; J age, 1993 [N dP IV]) La diminution de la résorption systémique est liée à l'action locale des vasoconstricteurs (l'adrénaline étant choisie comme référence) par stimulation des récepteurs alpha1 des muscles lisses des vaisseaux périphériques. La conséquence de cette action est la diminution de la perfusion tissulaire qui se traduit par l'ischémie locale des tissus (Allwood et coll, 1963 [Non classé]).

Cette ischémie concerne également les vasa nervorum qui alimentent les axones des fibres nerveuses sensitives concernées par l'anesthésie locale ; il s'en suit un ralentiss ement sensible du métabolisme des cellules nerveuses et donc de la transmission de l'influx nerveux ce qui aboutit à un approfondissement de l'anesthésie et à une augmentation de sa durée.

L'effet du vasoconstricteur comporte également une action facilitatrice sur la pénétration de l'AL dans la fibre nerveuse par stimulation directe de récepteurs adrénergiques anti-nociceptifs. Une telle action a été démontrée lors d'injections péridurales et intrathécales (Bromage et coll, 1983 [NdP IIb]; Yaksh et Reddy, 1981 [NdP Ib]).

Du point de vue clinique, le ralentissement de la vitesse d'absorption aboutit à deux résultats positifs démontrés de longue date :

(1) l'augmentation de la durée de l'anesthésie (V age, 1993 [NdP IV]);

(2) l'abaissement du pic plasmatique de l'AL, qui a, lui-même, deux conséquences bénéfiques : la diminution de la toxicité systémique et comme corollaire la possibilité d'augmenter la dose 
injectée. Covino et Vassallo (1976) [NdP V] ont ainsi rapporté que l'adjonction d'adrénaline permettait d'augmenter de $200 \%$ la durée d'une anesthésie à la lidocaïne à $0,5 \%$ tout en diminuant le pic plasmatique de $50 \%$. Le comportement des autres AL testés est voisin.

Notons toutefois que l'un des effets secondaires inattendus des vasoconstricteurs, lorsqu'ils sont associés aux $A L$, est de ralentir l'installation de l'anesthésie. Falaiye et Rood (1990) [NdP Ila] ont ainsi démontré que l'adjonction d'adrénaline à une solution de lidocaïne retardait sensiblement l'installation d'une anesthésie profonde appréciée au pulp-tester. Cet effet secondaire serait lié à la fois à un effet barrière $d u$ vasoconstricteur à l'égard de la solution anesthésique qu'il bloquerait sur le site d'injection, à distance de la fibre nerveuse visée, et à un effet acidifiant du vasoconstricteur par rapport au milieu qui est favorable au maintien de l'anesthésique sous sa forme non ionisée inactive.

L'association d'un vasoconstricteur à la solution anesthésique en odonto-stomatologie est donc indiquée parce que le vasoconstricteur diminue la résorption plasmatique du mélange injecté et assure ainsi une augmentation de la durée et de la profondeur de l'anesthésie tout en réduisant les effets systémiques de la solution.

\subsection{Les vasoconstricteurs peuvent-ils être utiles en pratique stomatologique en dehors de leur association à la substance anesthé- sique locale?}

Deux domaines de l'odonto-stomatologie font appel aux vasoconstricteurs en dehors de l'anesthésie :

- d'une part la prothèse : les cordonnets rétracteurs destinés à refouler la gencive au dessous des préparations de l'émail et/ou de la dentine lors de la prise d'empreinte (Pallasch, 1998 [NdP IV]);

- d'autre part : la chirurgie buccale et en particulier endodontique où parmi d'autres solutions l'injection de vasoconstricteurs ou la mise en place de supports en contenant a été préconisée pour limiter le saignement au centre de la zone chirurgicale (Syngcuk et Sivakami, 1997 [NdP IV]).

\subsubsection{Vasoconstricteurs sur les cordonnets de rétraction gingivale}

Les études concernant la toxicité des cordonnets de rétraction gingivale chargés de substance vaso-active (en pratique de l'adrénaline racémique à $8 \%$ ) sont contradictoires par la divergence des résultats toxicologiques et pharmacocinétiques.

Pour $3 \mathrm{~cm}$ de cordonnet Malamed (1997) [NdPIV] rapporte des valeurs allant de 225,5 à $661 \mu \mathrm{g}$ d'adrénaline racémique ce qui représente en réalité 113 à $330 \mu \mathrm{g}$ de la forme $\mathrm{L}$ pharmacologiquement active. Une telle quantité est équivalente selon Pallasch (1998) [NdP IV] à 3,13 à 9,16 catouches de $2 \mathrm{ml}$ de solution anesthésique dosée au 1/100000 d'adrénaline.

Les études concernant la tolérance ont montré chez le chien des élévations spectaculaires du rythme cardiaque et de la pression artérielle après mise en place de cordonnets. Le taux d'adrénaline plasmatique a été mesuré chez un seul patient passant de $15 \mathrm{pg} \cdot \mathrm{ml}^{-1}$ à $316 \mathrm{pg} \cdot \mathrm{ml}^{-1}$ après mise en place de cordonnets et ce sans effet hémodynamique.

Houston et coll (1970) [NdP IIb] se sont intéressés aux possibles effets hémodynamiques de ces cordonnets. Ils utilisent un protocole mal défini sur 9 sujets. Leurs résultats portent sur la pression artérielle et le rythme cardiaque à différents temps d'une prise d'empreinte. Ils démontrent des variations hémodynamiques négligeables.

D'autres travaux mettent en évidence une différence significative de la résorption systémique selon que l'épithélium gingival est intact ou qu'une gingivite est active ou encore qu'il est altéré par une préparation prothétique : l'épithélium créviculaire intact semblant constituer une barrière efficace au passage plasmatique de l'adrénaline.

II faut noter enfin que les différents protocoles sont difficilement comparables : durée de mise en place des cordonnets variant de 30 à 120 minutes, nombre de dents concernées variable, taille des cordonnets et dosages variables, non discrimination des quantités absorbées par le plasma, le fluide gingival et la salive.

Pallasch propose ainsi de retenir quelques règles concernant le cahier des charges d'une médecine buccale chirurgie buccale VOL. $9, \mathrm{~N}^{\circ}$ 2003 page 71 
médecine buccale chirurgie buccale recherche sur la tolérance des cordonnets chargés en adrénaline racémique : (1) taux d'adrénaline effectivement présente sur le cordonnet ; (2) durée de la présence du cordonnet dans le sulcus ; (3) état du sulcus ; (4) nombre de dents concernées ; (5) dilution par la salive et le fluide gingival ; (6) métabolisme de l'adrénaline racémique par les cathécol-0-méthyl transférases ; (7) vasoconstriction locale induite par l'adrénaline comme facteur diminuant sa propre absorption ; (8) traumatisme gingival lors de la pose des cordonnets ; $(9)$ sensibilité individuelle du patient à des variations même minimes $d u$ taux circulant de vasoconstricteur.

En l'absence de travaux concordants, il ne paraît pas possible de conclure quant à l'innocuité des cordonnets rétracteurs employés en prothèse dentaire. Les évaluations chez l'animal semblent montrer que les variations hémodynamiques sont inconstantes, et que leur caractère symptomatique est lié soit à une sensibilité du sujet, soit aux conditions expérimentales. La littérature ne rapporte qu'un seul incident grave chez l'homme (Hilley, 1984) [Non classé : cas clinique] lié à l'interaction halothane-adrénaline.

\subsubsection{Vasoconstricteurs comme moyen d'hé- mostase chirurgicale}

Les substances anesthésiques associées à des doses élevées de vasoconstricteurs ont été utilisées avec comme seul objectif de contrôler le saignement local par une infiltration large du site opératoire avant incision (Gutman, 1993 [NdP IV]). Les tenants de ces techniques font néanmoins observer que, d'une part, il est souvent difficile d'injecter à proximité des apex sans infiltrer des fibres musculaires squelettiques riches en récepteurs béta 2 adrénergiques qui sont responsables d'une vasodilatation plus que d'une vasoconstriction (Milam et Giovannitti, 1984 [NdP IV]) ; d'autre part, que cette vasoconstriction per opératoire est le plus souvent suivie d'une vasodilatation par hyperémie réactive : en effet, au fur et à mesure de la résorption du vasoconstricteur, celui-ci atteint sur le site chirurgical une concentration qui n'assure plus la stimulation des récepteurs alpha adrénergiques. Le flux sanguin retrouve alors rapide- ment son cours normal puis, par phénomène rebond, atteind progressivement un flux supérieur à la normale par réaction béta adrénergique : ce phénomène est lié à l'hypoxie locale des tissus et à l'acidose provoquée par la vasoconstriction prolongée. Lorsque cette hyperémie locale s'installe, les injections complémentaires d'AL avec vasoconstricteur sont sans effet (Gutman, 1993; Gutman et Harrison, 1994 [NdP IV] ; Syngcuk et Sivakami, 1997 [NdP IV]). L'adrénaline, la noradrénaline, la phényl-adrénaline ont été utilisées seules dans le contrôle du saignement local en chirurgie endodontique. Sommer (1962) [NdP IV] a utilisé, le premier, diverses solutions : adrénaline racémique à $1 / 1000$ et $1 / 500$, phényl-adrénaline à $1 / 100$ et noradrénaline à $1 / 200$, le support étant toujours la gaze; Ingle (1965) [NdP IV] proposait de combler la cavité osseuse avec de la gaze saturée d'une solution d'adrénaline racémique à $2 \%$ pendant 4 minutes. Grossman (1970) [NdP IV] préconise l'utilisation de rouleaux salivaires de coton imbibés d'adrénaline au $1 / 100000$ et propose également l'utilisation de pellets de coton chargés par une solution d'adrénaline racémique à 1,5\% .

Seules les quantités d'adrénaline présentes sur les pellets ont pu être évaluées, les autres techniques n'offrant aucune standardisation. Besner (1972) [NdP IIb] a pu ainsi démontrer que sur des pellets $n^{\circ} 2$ on trouve en moyenne $1,15 \mathrm{mg}$ d'adrénaline racémique sous forme d'hydrochlorure. L'application d'un pellet $n^{\circ} 2$ pendant 4 minutes n'a pas provoqué de variation de la fréquence cardiaque dans la série de Besner, ce que l'auteur attribue à la vasoconstriction locale provoquée par l'adrénaline et qui entraînerait une très faible et très lente absorption de l'adrénaline elle-même.

Notons qu'à côté des pellets chargés d'adrénaline seule, il existe également dans le commerce des pellets chargés à la fois d'adrénaline et de substances astringentes telles que le phénol sulfonate de zinc et le sulfate ferrique. Ces spécialités n'ont pas été testées dans la littérature et ne sont pas commercialisées en France à la connaissance du groupe de travail. Les techniques d'hémostase locale par emploi de vaso- 
constricteurs purs ou bien mélangés à des substances anesthésiques ou astringentes n'ont fait l'objet d'aucune évaluation publiée présentant un niveau de preuve satisfaisant. Elles sont donc du domaine empirique. Bien que largement diffusées, elles n'ont pas donné lieu à la publication d'accident ou d'incident en rapport avec les vasoconstricteurs.

\subsection{Les vasoconstricteurs peuvent-ils être associés aux anesthésiques généraux (AG) lors d'une anesthésie générale en odonto- stomatologe?}

L'utilisation de solution AL associées à un vasoconstricteur pour l'infiltration du champ opératoire au cours d'une anesthésie générale a été souvent préconisée comme moyen d'hémostase en chirurgie buccale et maxillo-faciale (Cantaloube et coll, 1991 [NdP IV]). II s'agit d'une alternative confortable et bactériologiquement sûre comparée aux manipulations destinées à fabriquer extemporanément un sérum adrénaliné. Le sérum adrénaliné est classiquement composé de chlorure de sodium à $9 \%$; l'action vasoconstrictrice étant obtenue par adjonction d'adrénaline en solution à $0,025 \%$ soit $0,25 \mathrm{mg}$ par ampoule de $1 \mathrm{ml}$ de sérum. Dans ces conditions, la dose usuelle d'adrénaline à ne pas dépasser serait de $0,01 \mathrm{mg} \cdot \mathrm{kg}^{-1}$ de poids du patient.

$L^{\prime} u t i l i s a t i o n$ d'un AL comme vecteur du vasoconstricteur outre les avantages de manipulation évoqués plus haut abaisse le seuil de l'analgésie, augmente la qualité du contrôle per et post opératoire de la douleur comme l'ont démontré Engquist et coll (1977) [N dP Ila], Hosoda et coll (1991) [NdP Ib], Yuge et coll (1995) [NdP IV], pour l'anesthésie péri-durale et surtout Mamiya et coll (1997) [NdP Ib] en chirurgie buccale.

Cantaloube recommande pour ces infiltrations soit le chlorhydrate de lidocaïne à $1 \%$ avec adrénaline au $1 / 100000$ ou à $2 \%$ avec adrénaline au 1/80 000 soit l'articaïne au 1/200 000 d'adrénaline soit la mépivacaiine avec noradrénaline au 1/100 000 .

Tordoff et coll (1996) [ $\mathrm{NdP}$ lb] ont cherché à savoir si l'utilisation d'une anesthésie locorégionale avant incision sous anesthésie générale pour l'avulsion de troisièmes molaires man- dibulaires diminuait la douleur post-opératoire. Ils ont injecté chez 36patients la même quantité de solution anesthésique de lidocaïne $2 \%$ adré nalinée à $1 / 200000$ d'un côté et une solution saline de l'autre. Le pourtour des dents extraites était également injecté. La douleur post opératoire était évaluée par échelle visuelle analogique. Aucune différence significative n'est apparue entre le sérum physiologique injecté et l'anesthésique.

Mamiya et coll (1997) [NdP Ib] ont pratiqué sur un groupe de 28 patients ASA 1 devant subir une ostéotomie sagittale mandibulaire bilatérale l'expérience suivante : les 28 patients ont été répartis en 4 groupes les groupes 1 et 2 ne recevaient pas d'anesthésie loco-régionale mandibulaire et se différenciaient par un niveau croissant de la profondeur de l'anesthésie générale par inhalation quantifiée par concentration alvéolaire minimale (MAC : minimum alveolar concentration) passant de 1,3 MAC à 1,6 MAC. Pour les groupes 3 et 4 les patients recevaient outre une anesthésie loco-régionale mandibulaire bilatérale, une anesthésie générale par inhalation respectivement 1,0 MAC et 1,3 MAC. II est à noter que les AL étaient identiques dans les deux groupes recevant l'anesthésie régionale : $4 \mathrm{ml}$ de mépivacaïne à 0,5\% . Dans les deux groupes 3 et 4 , une solution vasoconstrictrice différente était infiltrée localement (groupe $3: 8 \mathrm{ml}$ de lidocaïne à $1 \%$ additionnée de $10 \mu \mathrm{g} \cdot \mathrm{ml}^{-1} \mathrm{~d}^{\prime}$ adrénaline; groupe 4 : $8 \mathrm{ml}$ de propitocaïne à $3 \%$ additionnée de

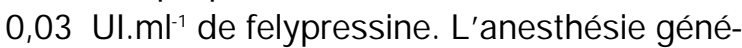
rale était réalisée avec les anesthésiques généraux suivants : induction par thiopenthal $\left(4 \mathrm{mg} \cdot \mathrm{kg}^{-1}\right)$ puis maintien par isoflurane et un mélange de protoxyde d'azote et d'oxygène à $40 \%$. Les contrôles des effets hémodynamiques de la chirurgie étaient les suivants : pression artérielle et fréquence cardiaque. La réponse sympathique était évaluée par dosage de la noradrénaline plasmatique. Les mesures commençaient à la $4^{\mathrm{e}}$ minute après infiltration locale et se répétaient au cours des différents temps chirurgicaux signific atifs.

Les résultats marquent des différences très significatives entre les groupes avec et sans médecine buccale chirurgie buccale VOL. $9, \mathrm{~N}^{\circ}$ 2003 page 73 
médecine buccale chirurgie buccale anesthésie loco-régionale. Aucune différence significative n'a été observée entre les groupes 3 et 4 . Les mêmes résultats s'observent quant au taux de noradrénaline plasmatiques qui sont significativement inférieurs dans les groupes avec anesthésie loco-régionale.

Les auteurs concluent donc que les infiltrations anesthésiques loco-régionales associées à l'infiltration de solution anesthésique additionnée d'un vasoconstricteur dans le site opératoire contribuent, d'une part, à diminuer et à prévenir la réponse sympathique endocrine autoimmune à l'agression chirurgicale qui est à l'origine d'une grande partie des douleurs post opératoires, d'autre part, à diminuer la profondeur de l'anesthésie générale nécessaire à l'intervention effectuée.

Une expérience voisine a été menée par Santoro et Marsicano (1998) [NdP Ilb], avec un but différent : vérifier les modifications hémodynamiques engendrées, cette fois, par l'injection d'adrénaline en rendant inopérants les facteurs de stress de l'état vigile par l'anesthésie générale. Les auteurs infiltraient 7 patients sous anesthésie générale avec une solution de $4 \mathrm{ml}$ de mépivacaïne à $2 \%$ adrénalinée à $1 / 100000$ au foramen mandibulaire. Les patients étaient soumis à des interventions de chirurgie buccale ou maxillo-faciale de nature très différentes. Les auteurs notaient les variations de la fréquence cardiaque et de la pression artérielle à différentes phases significatives de l'intervention.

Les auteurs concluent à partir d'un protocole très discutable que les variations du rythme et de la fréquence cardiaque observées lors d'une infiltration d'AL avec vasoconstricteur sous anesthésie générale sont inférieures à celles observées sous anesthésie locale à l'état vigile. L'utilisation d'une solution anesthésique additionnée d'un vasoconstricteur comme moyen de diminuer le saignement et d'abaisser le seuil de l'analgésie chez des patients opérés en chirurgie buccale sous anesthésie générale est une pratique couramment rapportée dans la littérature. Des expériences récentes menées en anesthésie péri-durale et tronculaire du nerf mandibulaire démontrent que cette pratique contribue à diminuer la réponse sympathique à l'agression chirurgicale et à diminuer la profondeur de l'anesthésie générale nécessaire. Une restriction doit être faite avec les AG volatils halogénés (halothane) qui ne doivent pas être utilisés avec l'adrénaline. Les AG halogénés provoquent en effet une potentialisation des effets dépresseur des catécholamines sur la vitesse de conduction des fibres de Purkinje du système autonome cardiaque (Camara et coll, 2001 [NdP Ilb]).

\section{Choix de la molécule vasoconstric- trice en odonto-stomatologie}

\subsection{Adrénaline versus noradrénaline}

L'adrénaline et les amines adrénergiques voisines provoquent la vasoconstriction en stimulant des récepteurs membranaires spécifiques des cellules des muscles lisses des vaisseaux. Deux types principaux de récepteurs adrénergiques alpha ${ }_{1}$ et alpha 2 peuvent initier la vasoconstriction. Anatomiquement, les récepteurs alphal sont situés près des nerfs sympathiques qui innervent les vaisseaux alors que les récepteurs alpha 2 sont disséminés de façon à répondre plus facilement aux catécholamines circulantes. La cascade des événements qui vont de la stimulation du récepteur à la vasoconstriction est maintenant bien établie (Ruffolo et coll, 1991 [NdP IV]).

Les récepteurs adrénergiques sont reliés à des enzymes effectrices et à des canaux ioniques par des protéines $\mathrm{G}$ c'est-à-dire des polypeptides qui fixent la guanosine triphosphate à l'instant où ces récepteurs sont stimulés par l'adrénaline. L'activation des protéines $G$ fixées aux récepteurs alpha ${ }_{1}$ adrénergiques provoque l'ouverture des canaux calciques de la membrane plasmatique et la stimulation d'une phospholipase $C$. Les ions calcium pénètrent alors dans la cellule et activent une kinase de la chaîne légère de la myosine calmoduline-dépendante. C'est elle qui, à son tour, initie la contraction musculaire. Pendant ce temps, l'hydrolyse de certains constituants de la membrane cellulaire par la phospholipase C conduit à la formation de diacylglycerol et d'inositol triphosphate. Ces seconds messagers indui- 
sent la contraction en facilitant la libération des réserves calciques intracellulaires et en entretenant l'activation de la protéine kinase $C$ qui contribue au support métabolique de la contraction. La stimulation par des vasoconstricteurs des récepteurs alpha ${ }_{2}$ ouvre également des canaux calciques par activation des protéines $G$. En outre l'adénylcyclase est inhibée par une protéine $\mathrm{G}$ spécifique inhibitrice.

Les récepteurs béta 2 adrénergiques activent au contraire l'adénylcyclase et provoquent par conséquent une vasodilatation. Les récepteurs béta $_{2}$ sont répandus dans les vaisseaux des muscles squelettiques et dans certains viscères, ils sont rare dans les muqueuses et la peau.

La noradrénaline partage avec l'adrénaline la capacité à stimuler les récepteurs alpha ${ }_{1}$ et alpha $_{2}$ mais elle $n$ 'interagit pas avec les récepteurs béta ${ }_{2}$ de sorte que le seul effet direct de la noradrénaline sur les vaisseaux est de favoriser leur constriction.

Ce caractère purement alpha-adrénergique a d'abord été mis à profit par l'industrie comme une preuve de spécificité de cette catécholamine. En fait, l'affinité de la noradrénaline pour les récepteurs alpha est moindre que celle de l'adrénaline ce qui implique l'utilisation de doses plus importantes de noradrénaline pour obtenir la même vasoconstriction (Knoll Kohler, 1988 [NdP IV]).

La noradrénaline est ainsi environ 4 fois moins vasoconstrictrice localement que l'adrénaline. La première conséquence en est une action plus courte par absorption plasmatique plus rapide. L'injection intravasculaire de noradrénaline a des conséquences plus sévères que celles de l'adrénaline : élévation de la pression artérielle systolique (> $200 \mathrm{~mm} \mathrm{Hg}$ ), augmentation de 75 à $80 \%$ de la pression artérielle moyenne et augmentation de la consommation myocardique en oxygène (Boakes, 1972 et 1973 [NdP IV]) ce qui en fait une molécule difficile à manipuler chez des patients souffrant d'ischémie myocardique. L'absence d'action de la noradrénaline sur les récepteurs béta ${ }_{2}$ produit une augmentation des résistances vasculaires périphériques qui explique largement sa toxicité. La noradrénaline possède en outre une action bradycardisante sévère et paradoxale car étant active sur les récepteurs cardiaques béta ${ }_{1}$, elle devrait provoquer en toute logique une accélération $d u$ rythme cardiaque. En fait, la noradrénaline pourrait également provoquer une stimulation réflexe des barorécepteurs aortiques et carotidiens en réponse à l'élévation des pressions diastoliques et systoliques et entraîner une bradycardie brutale (Berini et Gay, 1997 [NdP IV]). La durée de l'élévation de la pression artérielle consécutive à l'injection de noradrénaline est de 4 minutes et de 15 minutes pour l'effet bradycardisant (Knoll Kohler, 1988 [NdP IV]).

Des solutions anesthésiques additionnées d'une association d'adrénaline et de noradrénaline ont été commercialisées. Bien qu'aucune étude spécifique n'ait été publiée sur ce sujet, l'opinion de la littérature est peu favorable arguant que les effets bénéfiques de ces associations ne sont pas supérieurs à ceux de l'adrénaline seule alors qu'ils ajoutent les inconvénients de la noradrénaline ( age, 1993 [NdP IV], Berini et Gay, 1997 [NdP IV]).

\subsection{Autres vasoconstricteurs}

Devant les risques identifiés avec les catécholamines, les chercheurs et les industriels ont pensé à utiliser des substances vasoconstrictrices non catéchol dérivées d'une hormone naturelle sécrétée par la post-hypophyse : la vasopressine.

La felypressine (phényl alanine 2-lysine-vasopressine 8) est le chef de file des analogues de synthèse de la vasopressine, elle en possède les caractéristiques vasoconstrictrices locales sans en avoir les effets diurétiques puissants ni les effets vasoconstricteurs sur les artères coronaires (environ le tiers de l'action coronarienne de la vasopressine (Goldman et coll, 1971 [NdP Ilb]).

Elle n'agirait pas sur la pression artérielle ni sur le système nerveux central (Von Tsakiris et Bultmann, 1961).

Un travail récent et bien documenté (Sunada et coll, 1996 [NdP lla]) relativise les avantages systémiques de la félypressine qui fut considérée un temps comme le vasoconstricteur de choix chez les patients à antécédents d'ischémie myocardique (J ohnson et Widrich, 1977 [NdP lb]). Dans cet article, les effets myocardiques de différentes médecine buccale chirurgie buccale VOL. $9, \mathrm{~N}^{\circ}$ 2003 page 75 
médecine buccale chirurgie buccale

)L. $9, \mathrm{~N}^{\circ} 2$

2003

page 76 solutions de propitocaïne à $2 \%$ associées à des dosages variant de 0 (groupe témoin) à $+0,25 \mathrm{Ul}$. $\mathrm{ml}^{-1}$ de felypressine sont comparés chez 26 patients souffrant d'hypertension essentielle. Les résultats montrent que la pression systolique est augmentée par rapport au témoin pour les groupes à dosage élevés en felypressine mais que tous les groupes ont une élévation de la pression diastolique par rapport au témoin. Même si l'ischémie myocardique n'est pas mise en évidence de façon formelle en raison de biais expérimentaux, les auteurs observent une réduction de la contractilité myocardique dans les 3 groupes les plus dosés en félypressine. Ils concluent en recommandant chez les patients à hypertension essentielle une dose $0,18 \mathrm{UI}$ de felypressine ce qui correspond à $6 \mathrm{ml}$ de propitocaïne à $3 \%$ avec $0,03 \mathrm{UI}$ de felypressine.

Volpato (1999) [NdP Ib] montre qu'à dose élevée chez l'animal, la toxicité de l'adrénaline et de la fellypressine sont comparables mais que l'adrénaline aurait un effet «protecteur » quant à la survenue de convulsions lorsqu'elle est associée à la lidocaïne.

Shanks (1963) [NdP III] a démontré que les effets de l'interaction de la félypressine et des anesthésiques généraux halogénés sont voisins de ceux de l'adrénaline. Roberts et Sowray (1987) [Nd IV] recommandent de ne pas utiliser la félypressine chez la femme enceinte en raison d'une possible action inhibitrice sur la circulation placentaire par interférence avec le tonus utérin.

L'ornipressine (POR-8) est un autre analogue de synthèse de la vasopressine ayant des propriétés vasoconstrictrices locales. II a été un temps décrit comme un vasoconstricteur de choix en infiltration du champ opératoire mais des complications sévères (Kleemann et coll, 1986 [NdP IV]; Cantaloube, 1991 [NdP IV]) ont démontré son puissant effet constricteur sur les artères coronaires et l'ont fait abandonner.

Mélangée à un $\mathrm{AL}$ l'ornipressine a des performances très inférieures à l'adrénaline : il faut environ 10 minutes pour obtenir l'effet vasoconstricteur maximal (J age, 1993 [NdP IV]) ce qui en complique l'utilisation.

La corbadrine est encore trouvée en association avec l'aptocaïne, $\mathrm{AL}$ ayant connu un retour en grâce récemment du fait d'une bonne tolérance dans certaines porphyries hépatiques. La corbadrine est l'alpha-méthyl no radrénaline beaucoup moins toxique que la noradrénaline elle-même. Néanmoins son activité vasoconstrictrice est beaucoup plus faible. II faut donner des doses 10 fois plus fortes de corbadrine pour obtenir un effet comparable à celui de la noradrénaline. En outre, cette substance de synthèse est lentement éliminée d'où une prolongation de l'action qui peut constituer un obstacle. Cette prolongation de la durée d'action est liée à la présence du groupement méthyl qui empêche la dégradation de la corbadrine par la mono-oxydase. ( acquot et coll 1978 [NdP IV]).

L'adrénaline est industriellement et médicalement le chef de file des vasoconstricteurs utilisés seuls ou en association avec un $A L$ en odonto-stomatologie. Il dispose de la plus large casuistique qui confirme une grande sécurité de cette molécule. Les dérivés non catéchol n'ont pas fait la preuve de leur supériorité à ce jour même chez des patients susceptibles de mal tolérer les catécholamines.

\section{Indications des vasoconstricteurs en odonto-stomatologie}

Les qualités des vasoconstricteurs associés aux solutions d'AL ont été rappelées dans ce travail et justifient le très large emploi de ces associations lors des anesthésies par infiltration en odonto-stomatologie.

Le groupe de travail s'est interrogé à propos de deux techniques anesthésiques anatomiquement opposées pour lesquelles l'utilisation de vasoconstricteurs pose des problèmes différents. II s'agit :

- d'une part des anesthésies locales dites ponctuelles (anesthésie intra-pulpaire, intraseptale, intradiploïque et intraligamentaire) ;

- d'autre part les anesthésies loco-régionales au foramen mandibulaire qui sont effectuées dans un territoire richement vascularisé tant sur le plan artériel que sur le plan veineux ce qui élève le risque d'injection endovasculaire d'une substance anesthésique adrénalinée. 


\subsection{Les vasoconstricteurs doivent-ils être utilisés lors des anesthésies ponctuelles ?}

Les anesthésies ponctuelles ont en commun le fait que la solution anesthésique est infiltrée dans un espace anatomiquement clos où la diffusion sera minimale de sorte que la quantité injectée sera faible et que l'adjonction d'un vasoconstricteur a pu apparaître inutile voire nuisible en raison de l'agressivité tissulaire de la vasoconstriction locale qu'il provoque (Madrid et coll, 1991 [N dP IV]).

L'anesthésie intra-osseuse ou intradiploïque a fait l'objet de nombreuses études visant à étudier en particulier l'apport de ces techniques comme complément des techniques d'anesthésie locorégionales du nerf mandibulaire au foramen mandibulaire. Reitz et coll (1998) [NdP lb] ont testé une solution de $0,9 \mathrm{ml}$ de lidocaïne à $2 \%$ adrénalinée au 1/100 000 en injection intraosseuse au niveau des $2^{\text {e }}$ molaire, $1^{\text {re }}$ molaire et $2^{\mathrm{e}}$ prémolaire chez 38 sujets. Guglielmo et coll (1999) [NdP Ib] ont suivi un protocole strictement identique avec une solution de mépivacaïne à $2 \%$ additionnée de lévonordefrine à $1 / 20000$ chez 40 sujets. Dans les deux cas aucune complication locale n'a été rapportée par les auteurs à l'utilisation d'un vasoconstricteur en injection intra-osseuse. Dunbar et coll (1996) [NdP lb] et Coggins (1996) [NdP Ila] ont, quant à eux, relevé une inflammation et une suppuration au point d'injection mais dans les deux cas les auteurs incriminent la technique de perforation et non l'effet éventuel du vasoconstricteur.

Replogue et coll (1998) [NdP lb] a quant à lui établi la supériorité de l'injection intra-osseuse de lidocaïne à $2 \%$ adrénalinée à $1 / 100000$ par rapport à une solution à $3 \%$ de mépivacaïne sans vasoconstricteur lors de l'anesthésie de la $1^{\text {re }}$ molaire mandibulaire ce qui semble bien indiquer un intérêt dans l'usage de vasoconstricteurs puisque la mépivacaïne est pratiquement neutre pour la vasodilatation voire légèrement vasoconstrictrice. Ces résultats sont concordants avec ceux de Petrikas (1990) [NdP III] qui a étudié sur 22 sujets l'efficacité d'une solution de lidocaïne avec et sans adrénaline au cours d'une série d'injections intraseptales : il conclut que l'addition d'adrénaline apporte une amélio- ration de la profondeur de l'anesthésie, de son taux de succès et de sa durée, contribue à la dissémination pulpaire de l'anesthésie et n'augmente pas les effets délétères locaux.

Pour l'anesthésie intra-ligamentaire, les travaux de Tagger et coll (1994) [NdP Ib] ont clairement montré que l'anesthésie résulte plus d'une diffusion intra-osseuse que d'une diffusion directe jusqu'à l'apex de sorte que les remarques faites pour les techniques intra-osseuses devraient s'appliquer aux techniques intra-ligamentaires.

En fait, les travaux de Gray et coll (1990) [N dP Ila] ont montré à la fois l'absence d'effets délétères des vasoconstricteurs adjoints à la solution anesthésique injectée en intra-ligamentaire (lidocaïne + adrénaline ou prilocaïne + felypressine) et la supériorité marquée des taux de succès avec vasoconstric teurs par rapport aux AL seuls.

Les travaux de Walton (1982) [NdP Ilb] et de Galili et coll (1984) [NdP IIb] montrent un retour ad integrum du ligament desmodontal après 8 à 15 jours de cicatrisation chez le singe ayant subi des injections intra-ligamentaires de solutions comportant un vasoconstricteur. Tsirlis et coll (1992) [NdP lla] ont montré sur un groupe d'étude de 305 avulsions dentaires mandibulaires que la fréquence des alvéolites sèches après anesthésie intraligamentaire n'était pas augmentée par rapport à un groupe témoin avec anesthésié conventionnelle.

Mc Lean et coll (1992) [NdP Ib] ont démontré la supériorité d'une solution de bupivacaiine adrénalinée à 1/200 000 par rapport à une solution de lidocaïne adrénalinée à $1 / 100000$.

Enfin dans une étude méthodologiquement rigoureuse, Handler et Albers (1987) [NdP Ilb] ont comparé l'utilisation au cours des anesthésies intraligamentaires de 4 solutions différentes: lidocaïne à $2 \%$, lidocaïne $2 \%$ adrénalinée au $1 / 50000$, lidocaïne $2 \%$ adrénalinée au $1 / 100000$ et enfin adrénaline au 1/100000 seule. Leurs résultats surprenants montrent que contrairement à ce qui est habituellement rapporté, il n'existe pas de relation de proportionnalité entre la dose d'adrénaline présente dans la solution et la durée de l'anesthésie mesurée au pulp tester. II n'existe pas de différence entre les 4 solutions quant à la fréquence du succès médecine buccale chirurgie buccale VOL. $9, \mathrm{~N}^{\circ}$ 2003 page 77 
de l'anesthésie. L'anesthésie est aussi obtenue avec la solution d'adrénaline seule ce que les auteurs expliquent par le fait que l'anesthésie serait ici liée à la pression et non à l'action pharmacologique du vasoconstricteur.

L'utilisation d'un vasoconstricteur dans les techniques d'anesthésie ponctuelle n'est pas indispensable mais améliore considérablement la fréquence, la durée et la profondeur de l'anesthésie obtenue. Si l'injection est effectuée dans des conditions adéquates : pression contrôlée, injection lente, petits volumes $-0,2 \mathrm{ml}$ en moyenne par racine dentaire, (Handler et Albers, 1987) [NdP Ilb] - les lésions locales directement imputables au vasoconstricteur sont négligeables et réversibles. Les effets systémiques de ces injections existent mais sont le plus souvent très inférieurs à ceux observés dans les anesthésies par infiltration.

médecine buccale chirurgie buccale

L. $9, \mathrm{~N}^{\circ} 2$

2003

page 78

\subsection{Les vasoconstricteurs doivent-ils être utilisés lors des anesthésies loco-régionales du nerf mandibulaire?}

Pendant longtemps le risque d'une injection intravasculaire de solution anesthésique adrénalinée a été avancé comme argument en faveur de la proscription de l'emploi d'un vasoconstricteur dans la technique d'anesthésie au foramen mandibulaire (Gaudy et Aretto, 1999) [NdP IV]).

Cet argument s'oppose au sentiment consensuel dans la profession que le taux de succès de l'anesthésie loco-régionale mandibulaire est lié à la présence et au dosage du vasoconstricteur. La littérature ne confirme pas cette impression. Keesling et Hinds (1963) [NdP lb] ont comparé 5 solutions de lidocaïne $2 \%$ adrénalinées respectivement à $1 / 50000,1 / 250000,1 / 750000$, $1 / 1000000$ et sans adrénaline pour l'anesthésie au foramen mandibulaire. Les auteurs rapportent un taux de succès au pulp tester de $87,5 \%$ pour une durée moyenne d'anesthésie de $44 \pm 5,7$ minutes pour la lidocaïne sans adrénaline. A comparer par exemple avec un taux de succès de $96 \%$ et une durée moyenne de $66,9 \pm 8,7$ minutes pour la solution au $1 / 750000$. II ne semble pas exister de différence significative entre les solutions à $1 / 50000$ et $1 / 250000$, ni quant au taux de succès, ni quant à la durée de l'anesthésie.
Mac Lean et coll (1993) [NdP Ib] ont ainsi démontré en testant sur 30 sujets 3 solutions anesthésiques (lidocaïne $2 \%$ adrénalinée à $1 / 100000$, prilocaïne à $4 \%$ seule et mépivacaïne $3 \%$ seule) qu'il n'y avait pas de différence significative quant au taux de succès de l'anesthésie au foramen mandibulaire contrôlée au pulp tester.

Dagher et coll (1997) [NdP Ila] ont testé 3 solutions de lidocaïne à $2 \%$ adrénalinées respectivement à $1 / 50000,1 / 80000$ et $1 / 100000$. Selon la méthodologie de Mac Lean (1993) sur 30 sujets en bonne santé, les 3 solutions se révèlent équivalentes pour le taux de succès, le taux d'échec et la fréquence de l'anesthésie.

Malamed (1997) [NdP IV] ne recommande I'anesthésie tronculaire du nerf alvéolaire inférieur avec vasoconstricteur que dans le cas où une durée prolongée de l'anesthésie est recherchée.

Knoll-Kohler et Fortsch (1992) [NdP lb] ont testé, sur 10 étudiants, deux solutions de lidocaïne à $2 \%$ sans adrénaline, l'une de $\mathrm{pH} 3,5$, l'autre de $\mathrm{pH} 6,8$, et trois solutions de lidocaïne $2 \%$ adrénalinée au $1 / 50000$, au $1 / 100000$ et au $1 / 200000$. Selon ces auteurs la lidocaïne sans adrénaline quel que soit le $\mathrm{pH}$ présente un taux élevé d'échecs et une durée plus faible. L'adjonction d'adrénaline au 1/100000 ou au 1/200000 améliore le taux de succès et la durée de l'anesthésie mais ne fait pas varier le temps de latence. Ces résultats sont en accord avec ceux de Kabambe et coll (1982) [NdP Ib] qui observent, en comparant une solution de lidocaïne avec et sans adrénaline, un échec chez plus de la moitié de leurs sujets pour l'anesthésie du nerf alvéolaire inférieur.

L'addition d'un vasoconstricteur à la solution anesthésique n'est pas indispensable pour l'anesthésie au foramen mandibulaire du nerf alvéolaire inférieur. L'addition d'adrénaline augmente la durée de l'anesthésie mais ne semble pas avoir d'effet décisif sur le taux de succès. Les résultats concernant le taux de succès de l'anesthésie sont contradictoires. Compte tenu de la relation qui existe entre le taux de succès et le volume de solution injectée (Vreeland, 1989) [N dP IIb], I'addition d'un vasoconstricteur pourrait s'avérer judicieuse dans la prévention des effets systémiques des AL. 
Un autre argument invoqué pour contester l'utilisation d'un vasoconstricteur en anesthésie locorégionale du nerf mandibulaire est le fait que le vasoconstricteur pourrait allonger la durée de l'anesthésie labio-mentonnière et par conséquent favoriser les blessures par auto morsures labiales qui constituent l'un des effets indésirables les plus fréquents de ces injections (Wahl, 2000). En fait, des travaux ont démontré que seule l'anesthésie pulpaire est allongée au cours des anesthésies loco-régionales du nerf mandibulaire avec vasoconstric teur et non l'anesthésie labio-mentonnière (Hersh et Hermann, 1995 [Non classé : cas clinique]; Yaguiela, 1985 [NdP Ib]).

\section{Dosage des vasoconstricteurs en anesthésie odonto-stomatologique}

II n'existe pas de travaux définitivement concluants concernant le dosage idéal en adrénaline des $A L$.

Fink (1978) [NdP Ilb] a démontré que la durée de l'anesthésie locale (toutes techniques confondues) était directement dépendante de la dose d'adrénaline présente dans la solution : la durée obtenue pour une solution à $1 \%$ de lidocaïne adrénalinée au $1 / 50000$ est de 210 minutes, de 160 minutes au $1 / 100000$ et de 130 minutes au $1 / 200$ 000. Compte tenu de la durée moyenne des actes dentaires Himuro et coll (1989) proposent de remplacer la lidocaïne à 1/80000 par de la lidocaïne à $1 / 200000$ après un test comparatif sur seulement 6 volontaires. Dès 1967, Gangarosa et Halik [NdP lb] montraient que la solution de lidocaïne au 1/100000 ou au 1/300000 étaient équivalente pour la vitesse d'installation et l'efficacité évaluée en fonction de l'expérience d'un groupe de 17 chirurgiens dentistes au cours d'une série prospective en double aveugle de 542 patients. En ce qui concerne la durée de l'anesthésie, dont on estime depuis Braun (1924) [NdP III] qu'elle est dose-dépendante ; les résultats de la même étude montrent un gain négligeable en terme de durée en passant de la solution anesthésique adrénalinée au $1 / 300000$ à la solution adrénalinée au 1/100 000 . Knoll-Kohler (1992) [NP Ib] après étude chez 10 volontaires affirme que seule la lidocaïne à $1 / 100000$ donne des résultats constants.

Evidemment, les dosages de l'adrénaline doivent être adaptés aux caractéristiques de la molécule AL. En dehors de la lidocaïne, le marché est occupé par la carticaïne ou articaiine qui est commercialisée additionnée d'adrénaline au $1 / 100000$ et au $1 / 200000$.

Vahatalo et coll (1993) [NdP lb] ont testé la lidocaïne à $2 \%$ adrénalinée à $1 / 80000$ et l'articaine $4 \%$ adrénalinée au $1 / 200000$ pour le temps de latence et la durée de l'anesthésie contrôlée au pulp tester. II $n^{\prime}$ y avait pas de différence statistiquement significative entre les deux groupes ce qui permettrait de choisir la molécule la moins dosée en adrénaline.

Knoll-Kohler et coll (1992) [NdP lb] ont comparé l'articaine $4 \%$ à $1 / 100000$ et la solution à $1 / 200000$ lors d'une avulsion de $3^{e}$ molaire incluse mandibulaire en mesurant les variations de la fréquence cardiaque, la concentration d'AMP cyclique et le taux de noradrénaline. Ces paramètres furent corrélés aux taux plasmatiques d'adrénaline comme témoin de la sécrétion end ogène. La résorption d'adrénaline à partir du site d'injection s'est révélée dose dépendante ce qui aurait dû faire pencher les auteurs en faveur de la solution à 1/200000 d'adrénaline. En fait, dans un travail antérieur, Knoll-Kohler (1991) [NdP lb] estimait que le risque d'incident cardio-vasculaire était d'autant plus élevé que le geste opératoire était prolongé et que le dosage en ad rénaline était faible.

Ce résultat est contesté par les travaux de Daublander et coll (1997) [NdP III] qui constatent que les effets secondaires sympathicomimétiques des injections d'AL avec vasoconstricteur sont significativement supérieurs dans leur série rétrospective de plus de 2700 sujets pour la solution d'articaine à 1/100000 par comparaison avec la solution d'articaine au 1/200 000 .

J age (1993) [NdP IV] estime que la meilleure concentration chez le patient sain est (toutes molécules confondues) de $1 / 100000$ à $1 / 200000$ sachant que la dose maximale individuelle est de 0,25 mg. En revanche chez le patient présentant une affection vasculaire la concentration obligatoire serait de 1/200000. médecine buccale chirurgie buccale VOL. $9, \mathrm{~N}^{\circ}$ 2003 page 79 
médecine buccale chirurgie buccale
Rappelons que pour $1 \mathrm{ml}$ de solution anesthésique une solution à $1 / 1000$ représente $1000 \mu \mathrm{g}$ d'adrénaline tandis qu'une solution à $1 / 200000$ contient $5 \mu \mathrm{g}$ d'adrénaline.

Pour la mépivacaïne les travaux de Berling (1958) [N dP Ilb] montrent une absence de différence statistiquement significative entre les solution à $2 \%$ adrénalinées respectivement à $1 / 100000$ et $1 / 200000$ pour le taux de succès et la durée de l'anesthésie pulpaire.

Les travaux sont encore contradictoires concernant le dosage idéal de l'adrénaline dans les solutions à $2 \%$ de lidocaïne. La solution à $1 / 200000$ donne plus de deux heures d'anesthésie ce qui représente une durée suffisante pour l'immense majorité des actes odonto-stomatologiques. Pour l'articaine à $4 \%$ et la mépivacaïne à $2 \%$, les solutions au $1 / 200000$ devraient être préférées en l'absence de différence significative des performances avec la solution au 1/100000.

Enfin les travaux de J orkjend et Skoglung (2000) [NdP lb] démontrent clairement :

- d'une part que l'augmentation du volume de l'adrénaline et de la solution anesthésique locale injectée peut avoir un effet indésirable qui est l'accroissement de la douleur post-opératoire sans contreparties en terme de durée ou de qualité de l'anesthésie ;

- d'autre part que l'augmentation du dosage de l'adrénaline dans la solution anesthésique accroit également significativement la douleur post-opératoire par élévation du taux d'AMP cyclique dans les tissus gingivaux ce qui favorise l'accumulation de substances nociceptives ou de médiateurs pro-algésiques.

\section{Interactions médicamenteuses}

Les vasoconstricteurs employés en association avec les $A L$ mais aussi comme hémostatiques topiques ou injectables et enfin sur les cordonnets de rétraction gingivale ont le potentiel d'interagir avec une large variété de drogues (Hansten, 1981) [NdP IV].

Les réactions locales vont jusqu'à l'ischémie locale et à la nécrose (Yagiela, 1999 ; Damm et
Fantasia, 1992). Elles sont liées au surdosage relatif par saturation des tissus en vasoconstricteur et à l'injection trop rapide (M eechan, 1998) La plupart des réactions systémiques sont de courte durée en raison principalement de la rapide inactivation des vasoconstricteurs une fois qu'ils sont absorbés dans le courant sanguin (Yaguiela, 1999) [NdP IV]. Néanmoins des lésions graves voire le décès du patient peuvent résulter d'une fibrillation d'origine médicamenteuse, d'un infarctus du myocarde ou d'un accident céréb rovasculaire (Hilley, 1984; Okada, 1989; Massalha, 1996) [Non classés : cas cliniques].

\subsection{Antidépresseur tricycliques}

IIs sont progressivement remplacés par des inhibiteurs sélectifs de la recapture de la sérotonine, ils restent néanmoins utilisés chez les patients intolérants ou résistants à ces drogues nouvelles. Les antidépresseurs tricycliques bloquent la recapture active des neurotransmetteurs amines biogènes (catécholamines et sérotonines) par les terminaisons nerveuses où elles ont été relarguées. Le résultat est une potentialisation des neurotransmetteurs concernés : les vasoconstricteurs adrénergiques mais surtout la noradrénaline sont sujets au même phénomène de recapture. Les antidépresseurs tricycliques bloquent les récepteurs muscariniques et alpha adrénergiques et dépriment le myocarde ce qui peut en retour modifier la réponse cardiovasculaire aux vasoconstricteurs.

Selon Boakes et coll (1972) [NdP IV] sur 15 cas de patient ayant présenté des troubles sévères à la noradrénaline, 5 prenaient des antidépresseurs tricycliques.

De tels accidents peuvent se produire pour des injections de 2,5 cartouches d'adrénaline à 1/100000 (Persson et Siwers, 1975) [N dP IV]. Cawson et coll (1983) [NdP IV] ont écarté la possibilité d'interactions cliniquement significatives entre antidépresseurs tricycliques et $\mathrm{AL}$ contenant de l'adrénaline.

En réalité si le risque théorique est élevé les manifestations cliniques sont rares. Plusieurs facteurs y contribuent :

- la compétition entre l'effet vasoconstricteur ( $\alpha$ adrénergique) et vasodilatateur ( $\aleph_{2}$ adréner- 
gique) aboutit à une compensation des variations hémodynamiques aux doses usuelles en odonto-stomatologie ;

- la prescription de ces drogues se fait souvent au long cours ce qui provoque une désensibilisation aux vasoconstricteurs adrénergiques et par voie de conséquence une diminution du risque d'interaction (Moyer et coll, 1979) [NdP Ib].

L'attitude face aux patients sous antidépresseurs tricycliques doit être d'écarter la noradrénaline en association aux $A L$ et d'injecter des doses mesurées d'AL associé à de l'adrénaline au $1 / 100000$ ou au $1 / 200000$. En pratique la dose injectée devrait être le tiers de la dose maximale chez le sujet normal (Yaguiela, 1999 [NdP IV]).

\subsection{Inhibiteurs de la monoamine-oxydase}

Les inhibiteurs sélectifs seuls encore utilisés ne présentent pas d'interaction avec l'adrénaline. Des études répétées chez l'Homme et l'animal n'ont montré aucune interaction significative aux doses utilisées en odonto-stomatologie (Boakes et coll, 1973 [NdP IV]; Wong et coll, 1980 [NdP lb]).

\subsection{Béta-bloquants}

Bien que des effets broncho et vasoconstricteurs puissent théoriquement se manifester lors de la prise de béta-bloquants cardio ou béta ${ }_{1}$ sélectifs (qui sont ainsi susceptibles de provoquer des crises d'asthme), il n'existe aucun incident décrit chez des patients chez lesquels ont été associés béta-bloquants, cardio-sélectifs et solution anesthésique adrénalinée (Pallash, 1998 [NdP IV]).

Ce sont donc principalement des béta-bloquants non cardio-sélectifs qui bloquent de façon compétitive la stimulation des récepteurs béta $_{1}$ et béta 2 par les catécholamines endogènes qui sont en cause. Ils bloquent aussi l'activation des récepteurs béta par des catécholamines exogènes. C'est principalement au niveau des récepteurs béta 2 que les béta-bloquants vont agir en transformant l'adrénaline en une drogue exclusivement alpha-adrénergique. Les conséquences en sont une augmentation des résistances périphériques et, directement en rapport avec la dose, une augmentation de la pression artérielle et un ralentissement de la fréquence cardiaque qui peuvent déboucher sur des accidents majeurs et bien documentés (Hansbrough et Near, 1980; Foster et Aston, 1983 [Non classés : cas cliniques]).

Ce risque doit être tempéré pour les béta-bloquants non cardio-sélectifs avec activité sympathomimétique intrinsèque (ASI) pour lesquels l'activité béta-agoniste partielle aboutit à une limitation de l'effet bradycardisant et vasoconstricteur.

Notons que des effets locaux bénéfiques ont été décrits pour les béta-bloquants comme l'allongement de la durée de l'anesthésie pulpaire et des tissus mous (Zhang, 1999 [NdP lb]).

Les précautions comprendront une injection fractionnée et lente d'un AL additionné d'adrénaline au maximum au $1 / 100000$ après aspiration négative.

\subsection{Anesthésiques généraux}

Nous avons vu les interactions possibles avec les dérivés halogénés.

Le mécanisme par lequel les AG potentialisent les effets arythmogènes des catécholamines sont inconnus. II s'agit probablement d 'une stimulation simultanée des récepteurs alpha ${ }_{1}$ et béta. L'adrénaline est à son tour capable d'activer les deux types de récepteurs et d'engendrer des troubles du rythmes pendant l'anesthésie générale (Hayashi, 1993 [NdP lb]).

Le thiopental est également capable d'exagérer le potentiel arythmogène des substances adrénergiques. Etant donné que le thiopental est souvent utilisé à l'induction avec des dérivés halogénés, son interaction a d'abord été sous estimée.

Lorsque le thiopental est utilisé seul, un dosage d'adrénaline de $2 \mu \mathrm{g} . \mathrm{kg}^{-1}$ sera admis en injection per opératoire sous anesthésie générale. Ce dosage sera de $1 \mu \mathrm{g} \cdot \mathrm{kg}^{-1}$ si le thiopental est associé à de l'halothane (Christensen et coll, 1993 [NdP IV]). On a rapporté une mort sous halothane, due à une interaction avec un cordonnet rétracteur à l'adrénaline racémique (Hilley et coll, 1984 [Non classé : cas clinique]).

\subsection{Cocaïne}

Des expériences chez l'animal et des cas humains ont apporté la preuve de l'interaction de médecine buccale chirurgie buccale VOL. $9, \mathrm{~N}^{\circ}$ 2003 page 81 
médecine buccale chirurgie buccale la cocaïne avec les vasoconstricteurs adrénergiques. (Lathers et coll, 1988) [Non classé : cas clinique]. Plusieurs décès dont l'étude est bien documentée, figurent dans la littérature (Chiu, 1986) [Non classé : cas clinique]. Le mécanisme est un effet facilitateur de la cocaïne sur le relargage des neurotransmetteurs adrénergiques et l'intensification des réponses post synaptiques aux substances adrénalino-mimétiques. Le blocage des récepteurs muscariniques cardiaques et l'altération centrale du système nerveux autonome peuvent en outre contribuer à aggraver les réactions à l'injection de vasoconstricteurs.

Aucun traitement dentaire ne doit être effectué chez un patient sous l'empire de stupéfiants. Les vasoconstricteurs seront proscrits au moins 24 heures après la consommation de la cocaïne pour permettre l'élimination de la drogue et de ses métabolites actifs.

\subsection{Antipsychotiques et alpha-bloquants}

Ces médicaments (chlorpromazine, thioridazine, risperidone) ont pour effet secondaire de bloquer les récepteurs alpha-adrénergiques et donc de provoquer des réactions d'hypotension orthostatique. En cas de surdosage, le passage plasmatique d'adrénaline serait aggravant puisque seuls les récepteurs béta ${ }_{2}$ seraient activés entraînant une vasodilatation.

II s'agit en fait d'un risque strictement théorique: aucun accident sérieusement documenté n'a été rapporté aux doses utilisées en odontostomatologie.

\subsection{Guanethidine}

S'agissant d'une substance inhibant le relargage de la noradrénaline au niveau des fibres terminales sympathiques, elle est utilisée dans le traitement de l'hypertension artérielle sévère: utilisée au long cours elle pourrait susciter une multiplication des récepteurs adrénergiques ou un abaissement de leur seuil de sensibilité.

Ce risque est théorique pour un méd icament rare.

\subsection{Anorexigènes adrénergiques}

Ce sont des drogues sympathomimétiques qui affectent le métabolisme des catécholamines et sont chimiquement voisines des amphéta- mines. Ces drogues accroissent la neurotransmission adrénergique et stimulent le système nerveux central, leur activité anorexigène découle de cette stimulation du SNC.

Leurs effets peuvent être potentialisés par l'utilisation concomitante de substances vasoconstrictrices. C'est le cas du mazindol pour lequel la FDA et le fabriquant recommandent des précautions d'emploi avec les vasoconstricteurs (Wynn, 1997 [NdP IV]).

\section{Existe-t-il des pathologies contre- indiquant les vasoconstricteurs?}

On doit avoir à l'esprit que $45 \%$ des consultants d'un cabinet dentaire ont une ou plusieurs pathologies intercurrentes à la pathologie bucco-dentaire et que $20 \%$ d'entre eux présentent une affection cardio-vasculaire. Daublander et coll (1997) [NdP III] estiment dans leur série à $0,07 \%$ le risque d'accident grave en rapport avec l'anesthésie locale au cabinet dentaire ce qui est comparable à l'incidence des complications graves de l'anesthésie générale $(0,05 \%)$. $M$ ais ces chiffres doivent être pondérés par le statut du patient. Ainsi les effets secondaires observés sous anesthésie locale dentaire concernent $5,7 \%$ des patients à risques contre $3,5 \%$ des patients sains. Ces chiffres sont à rapprocher de ceux de l'anesthésie générale pour laquelle les effets secondaires affectent $12,3 \%$ des patients ASA I et $34,9 \%$ des patients ASA III et IV. Daublander montre clairement que les effets secondaires relevés sont indépendants de la molécule anesthésique utilisée (articaine, lidocaïne ou mépivacaïne) et de la présence ou non de vasoconstricteur associé mais dépendent plutôt du dosage employé.

L'anesthésie locale avec vasoconstricteur est donc, en odonto-stomatologie, une technique d'une grande sécurité dont les contre-indications paraissent largement exagérées.

\subsection{Hyperthyroïdie}

L'hyperthyroïdie peut résulter d'une maladie ou découler d'un surdosage chronique en thyroxine. L'hyperthyroïdie se traduira par des 
troubles cardiovasculaires qui reproduisent les effets d'un surdosage en adrénaline : tachycardie et autres arythmies, élargissement de l'amplitude du pouls, ischémie myocardique... On a longtemps cru que l'adrénaline et la noradrénaline participaient aux désordres de l'hyperthyroïdie de façon synergique avec la thyroxine; II n'en est rien : en cas d'hyperthyroïdie, les réponses hémodynamiques à l'action de l'adrénaline et de la noradrénaline ne sont pas fondamentalement changées. (Yaguiela, 1999 [NdP IV]).

Des études récentes (V ohnson, 1995 [NdP Ib]) ont démontré que les patients hyper et hypothyroïdiens n'ont pas de troubles majeurs lorsqu'ils sont soumis à un traitement correcteur et mis en présence de catécholamines à distance du début de ce traitement. Bien que le risque théorique de potentialisation thyroxine-adrénaline soit sérieux, il n'existe pas de cas clinique rapporté.

\subsection{Hypertension}

L'hypertension et sa relation aux vasoconstricteurs font l'objet d'une abondante littérature. II est maintenant largement admis que le passage plasmatique du vasoconstricteur est pratiquement négligeable en termes d'effet cardiovasculaire (augmentation de la fréquence cardiaque et de la pression artérielle) en comparaison de la sécrétion de catécholamines endogènes lors de la douleur et du stress.

La totalité du débat sur les changements cardio-vasculaires induits par l'adjonction éventuelle d'un vasoconstricteur à l'AL tourne donc autour de l'évaluation de l'apport exogène (une vingtaine de $\left.\mu \mathrm{g} . \mathrm{l}^{-1}\right)$ provoqué par l'injection d'une carpule en comparaison du taux plasmatique courant qui est autour de $300 \mu \mathrm{g} \cdot \mathrm{I}^{-1}$. Si I'on s'interroge sur la capacité physiologique à absorber un tel apport exogène, certains faits bien documentés peuvent être pris en compte: lors de l'accouchement sans péridurale les douleurs du travail peuvent multiplier par un facteur de 4 à 6 le taux d'adrénaline plasmatique (Bonica, 1999 [NdP IV]); lors d'une extraction dentaire le seul stress sans douleur multiplie l'adrénaline plasmatique par un facteur 10 ou 20. Le passage plasmatique lors de la résorption de $2,2 \mathrm{ml}$ de lidocaïne adrénalinée à $1 / 100000$ provoque seulement un doublement du taux de l'adrénaline plasmatique (Tolas, 1982 [NdP Ila]).

Chernow et coll (1983) [NdP Ib] comparent l'injection d'AL associé à de l'adrénaline et sans vasoconstricteur chez 14 sujets sains pour les variations de la pression artérielle, du rythme cardiaque et du dosage des catécholamines endogènes. Ils relèvent des résultats significatifs après injection de la solution adrénalinée pour l'élévation du taux de catécholamines pendant 60 minutes après injection: le taux passant de $27 \pm 4 \mathrm{pg} . \mathrm{I}^{-1}$ à $94 \pm 13 \mathrm{pg} . \mathrm{I}^{-1}$ dans un rapport de 1 à 3. La même équipe (Cioffi, 1985 [NdP IIb]) rapporte une augmentation dans un rapport de 1 à 3,5 dans le taux des catécholamines circulantes après anesthésie adrénalinée pour soins conservateurs. Ces taux se trouvant divisés par deux dès le début de la procédure de soins (pose de la digue).

En 1993, Lipp et coll [NdP Ib] tentent d'isoler dans l'augmentation des catécholamines qui suit une injection anesthésique la part qui résulte de l'apport exogène par la solution anesthésique avec vasoconstricteur de la part qui résulte de l'élévation des catécholamines endogènes liée au stress de l'infiltration et à la peur globale de l'acte opératoire.

Pour cela les auteurs utilisent une injection d'articaine à $4 \%$ additionnée de $20 \mu \mathrm{g}$ d'adrénaline marquée. Ils observent une élévation de l'adrénaline totale (exogène et endogène) de 5 à 10 fois supérieure par rapport au groupe témoin traité sans anesthésie. Ils observent également deux pics sériques de l'adrénaline: un 5 minutes après le début de l'injection, l'autre après le début du traitement dentaire. Les auteurs affirment que l'augmentation des catécholamines totales est principalement le reflet de l'apport exogène et expliquent le pic qui marque le début des soins par l'accroissement du taux de la résorption plasmatique de l'anesthésique liée au massage inévitable du site d'injection par les doigts de l'opérateur.

Niwa et coll (2000) [NdP Ib] dans un travail particulièrement détaillé et sophistiqué ont établi médecine buccale chirurgie buccale VOL. $9, \mathrm{~N}^{\circ}$ 2003 page 83 
médecine buccale chirurgie buccale

)L. $9, \mathrm{~N}^{\circ} 2$

2003

page 84 que l'infiltration de $3,6 \mathrm{ml}$ de lidocaïne $2 \%$ adrénalinée au 1/80000 était équivalente, en termes d'effets hémodynamiques et de taux plasmatiques de catécholamines, à la perfusion de $10 \mathrm{ng} \cdot \mathrm{kg} \cdot \mathrm{mn}^{-1}$ d'adrénaline seule. Le taux plasmatique de catécholamines passant de $52 \pm 24$ pg..$^{-1}$ à $363 \pm 105$ pg..$^{-1}$ pour l'infiltration anesthésique adrénalinée et de $32 \pm 18$ pg..$^{-1}$ à $214 \pm 69 \mathrm{pg} . \mathrm{l}^{-1}$ pour la perfusion de $10 \mathrm{ng} \cdot \mathrm{kg} \cdot \mathrm{min}^{-1}$, c'est-à-dire se trouvant multiplié par un facteur 7 .

Mais si l'on a pu démontrer une augmentation significative des catécholamines totales après injection d'AL avec vasoconstricteur et même démontrer la part prépondérante des catécholamines exogènes dans cette augmentation, il est impossible de connaître avec précision le retentissement cardio-vasculaire de cette augmentation car d'autres facteurs surajoutés rendent l'interprétation des résultats aléatoire. Ainsi, Di Angelis et Luepker (1983) [NdP Ila] ont démontré que le seul fait de se rendre chez le chirurgien dentiste pour un simple contrôle provoquait une élévation de la pression artérielle supérieure de $4,5 \mathrm{~mm} \mathrm{Hg}$ par rapport à une visite de contrôle chez le médecin. Les travaux de Gortzack et coll (1992) [N dP Ib] confirment ces résultats et montrent qu'il n'y a aucune différence entre sujets normo et hypertendus sur ce point. De nombreux auteurs enfin estiment qu'il est impossible d'établir un lien de proportionnalité entre augmentation des catécholamines totales et effets cardio-vasculaires, puisque si la plupart observent cette augmentation après injection d'AL avec vasoconstricteur, ils s'accordent pour ne pas observer chez des sujets sains ou hypertendus de retentissement clinique manifeste (Sack et Kleeman, 1992 [NdP lla]).

Cheraskin et coll (1958) [NdP lb] ont de leur côté démontré que l'essentiel de l'élévation de la pression artérielle avait lieu en salle d'attente et non à la phase opératoire. Cette élévation de la pression artérielle n'était pas significativement différente entre hypertendus et normotendus mais elle était significativement réduite chez les sujets hypertendus qui recevaient une prémédication sédative 45 minutes avant I'heure du rendez-vous. Ce travail mettait ainsi clairement en évidence le rôle du stress par rapport au rôle discret de l'injection de catécholamines exogènes lors de l'AL.

Les mêmes auteurs ont démontré lors de travaux ultérieurs (Cheraskin et Prasertsuntarasai, 1959 [N dP lb]) que la phase des 5 à 10 minutes qui suivent l'injection anesthésique avec ou sans adrénaline n'est marquée par aucun changement significatif de la pression artérielle ou du pouls, que le patient soit initialement normo ou hypertendu. Ils annoncent les résultats ultérieurs de Campbell (1996) [NdP Ilb] en précisant que c'est au début et à la fin de l'acte opératoire lui même que les changements les plus significatifs sont observés. Leur conclusion est que l'utilisation chez les hypertendus d'un séd atif préopératoire et d'un vasoconstricteur associé à l'AL donne des résultats signific ativement supérieurs en terme de contrôle de la pression artérielle et du pouls comparés aux hypertendus qui ne reçoivent ni sédatif ni vasoconstricteur. Ce résultat est à rapprocher des travaux de Goldstein (1982) [N dP lb] qui montrent que les variations hémodynamiques observées sont principalement dues à la sécrétion de nor-adrénaline endogène sous l'effet du stress opératoire, sécrétion bien compensée par l'administration d'une prémédication sédative par diazépam.

Meyer (1987) [N dP lb] a comparé les variations de la pression artérielle chez 60 sujets sains recevant pour 30 d'entre eux une injection $A L$ adrénalinée et pour les 30 autres une injection $\mathrm{AL}$ sans vasoconstricteur. Les résultats montrent que la pression artérielle des patients du groupe sans vasoconstricteur sont significativement plus mauvais, en terme de contrôle de la pression artérielle, que ceux du groupe avec. L'auteur explique logiquement ces résultats par la mauvaise qualité de l'anesthésie obtenue lorsque l'AL était injecté seul et donc par l'hypersécrétion de catécholamines endogènes. II est à noter que dans les deux groupes la moitié des sujets subissaient seulement l'anesthésie tand is que les autres étaient soumis à une anesthésie suivie par une avulsion dentaire. Dans le groupe sans vasoconstricteur, ceux qui subissaient l'avulsion présentaient les variations significatives de la pression artérielle. 
Il n'y a donc pas de contre-indication à utiliser un $\mathrm{AL}$ associé à de l'adrénaline en particulier pour des actes nécessitant une anesthésie locale prolongée et profonde chez un sujet hypertendu stabilisé par le traitement antihypertenseur.

Les doses maximales recommandées sont de $0,04 \mathrm{mg}$ au total ce qui correspond à 2 cartouches ou 4,4 $\mathrm{ml}$ d'AL adrénaliné à 1/100000. (Budentz, 2000 [NdP IV]).

Pour la grande majorité des hypertendus équilibrés, il sera possible de dépasser cette limite en tenant compte des règles de surdosage (Meechan, 1998 [NdP lb]). Chez les patients déséquilibrés sur le plan tensionnel, on pourra poursuivre l'anesthésie au-delà des deux cartouches adrénalinées avec un anesthésique sans vasoconstricteur.

Massalha et coll (1996) [Non classé : cas clinique] rapportent 2 cas d'hémorragies intracérébrales ayant entraîné la mort du patient au cours de soins dentaires. Nous les citons pour mémoire. Ils effectuent une revue de la littérature et mettent, comme la plupart des auteurs, ces poussées hypertensives majeures aboutissant à la mort sur le compte d'une hyperstimulation du trijumeau.

Le ganglion trigéminal, en effet, outre la sensibilité de la face et la motricité masticatrice, assure l'innervation vasomotrice de tous les vaisseaux sanguins cérébraux (Moskowitz, 1984 [NdP IV]). Ils concluent à un effet simultané de cette hyperstimulation et des changements hémodynamiques entraînés par l'élévation du pic sérique des catécholamines après injection anesthésique. Leur point de vue est purement conjectural.

En cas d'instabilité tensionnelle associée à d'autres éléments grevant le pronostic, les soins devront être menés en milieu hospitalier disposant d'une structure de réanimation et effectués sous monitorage.

\subsection{Troubles du rythme}

La littérature concernant l'étude des variations du rythme cardiaque induites par l'injection d'un AL avec vasoconstricteur est riche et souvent d'un très bon niveau de preuve (Meyer, 1987 [NdP Ib]; Montebugnoli, 1990 [NdP lb];
Blinder, 1996 [NdP Ilb]; Campbell, 1996 [NdP Ilb]; Replogue, 1999 [NdP lb]).

Un travail très bien documenté de Campbell et coll (1996) [N dP Ilb] a permis d'étudier les variations du rythme cardiaque dans une population de 40 sujets âgés (20 témoins et 20 arythmiques (traités ou non)) à différents temps de I'intervention: pré-opératoire, injection anesthésique, per-opératoire, post-opératoire. Les résultats permirent de faire tomber quelques lieux communs: (1) il n'y eut aucune différence significative entre le groupe témoin et le groupe arythmique pour l'installation d'un épisode d'arythmie bénigne (17 sujets); (2) les résultats montrent de façon paradoxale que l'élévation du rythme cardiaque est nettement inférieure au cours de l'injection anesthésique comparée à l'élévation pré et per opératoire du rythme cardiaque; (3) les auteurs démontrent en outre que l'injection de solution anesthésique avec vasoconstricteur n'est probablement pas impliquée dans les épisodes d'arythmie bénigne observés: le pic sérique du vasoconstricteur était atteint en moyenne 5 minutes après l'injection alors que les épisodes d'arythmie se sont situés à la moitié du temps chirurgical, c'est-à-dire à distance du pic sérique en vasoconstricteur.

Ce travail est à rapprocher des résultats de Blinder et coll (1996) [NdP Ilb] sur les changements électrocardiographiques observés par holter chez 40 patients cardiaques suivis 1 heure avant une avulsion sous AL sans vasoconstricteur et 23 heures après. Ces résultats montrent que 14 patients ont présenté des changements significatifs à l'ECG et 12 d'entre eux une arythmie alors même qu'aucun vasoconstricteur n'était présent dans la solution.

Un autre résultat est que 12 des 14 patients ayant présenté un changement significatif à I'ECG étaient sous digoxine, soit pour des accidents ischémiques soit pour fibrillation auriculaire. Ces résultats semblent démontrer la capacité de l'injection anesthésique seule à provoquer, via le stress physiologique et psychologique qui lui sont associés et via l'interaction des effets pro-arythmogènes des $A L$, des modifications aiguës de la fréquence cardiaque chez un pourcentage significatif de patients médecine buccale chirurgie buccale VOL. $9, \mathrm{~N}^{\circ}$ 2003 page 85 
médecine buccale chirurgie buccale

)L. $9, \mathrm{~N}^{\circ} 2$

2003

page 86 qu'ils soient sains ou non sur le plan cardio-vasculaire (Malamed, 1996).

Les auteurs rejoints par Stanley Malamed [NdP IV] recommandent la monitorisation des patients sous digoxine lors d'une anesthésie locale.

Les injections intra osseuses qui provoquent une élévation plus importante du rythme cardiaque et de la pression artérielle doivent être évitées (Chamberlain, 2000 [NdP IIb]). Replogue et coll (1999) [NdP Ib] proposent l'utilisation d'une solution à $3 \%$ de mépivacaïne comme alternative à l'injection d'une solution AL adrénalinée chez ces patients.

Les troubles du rythmes rencontrés en pratique courante sont pour l'essentiel des fibrillations auriculaires équilibrées par un traitement adapté (Anguera Camos et Brugada Terradellas, 2000 [NdP IV]). Dans ces conditions le contrôle du stress et de la fréquence cardiaque thérapeutique est essentiel et l'utilisation d'anesthésiques avec vasoconstricteurs est indiquée. Les règles de dosage sont les même que précédemment.

\subsection{Cardiopathies coronariennes}

Cette pathologie est fréquemment liée aux deux précédentes et il paraît évident que des réponses voisines peuvent être faites, néanmoins compte tenu de l'extrême fréquence de cette pathologie et de la confusion qui existe dans les esprits à leur sujet, tant chez les médecins généralistes ou spécialistes que chez les chirurgiens dentistes, le groupe de travail a tenu à lui consacrer un paragraphe spécifique.

Les ischémies myocardiques épisodiques au cours des coronaropathies stables ou instables connues passent cliniquement inaperçues dans plus de 2/3 des cas (Quyyumi et coll, 1985 [NdP Ila]). La chirurgie extra cardiaque, même mineure, est une des sources identifiées d'épisodes d'ischémie myocardique chez les coronariens connus (Deanfield, 1984 [N dP Ila]).

De nombreux travaux ont montré que les anomalies du segment ST de l'ECG traduisent des épisodes d'ischémie myocardique. En 1989, Vanderheyden et coll [ $\mathrm{NdP} \mathrm{Ib}$ ] ont étudié ces anomalies du segment ST au cours de soins parodontaux sous $A L$ avec vasoconstricteur chez des patients coronariens connus et traités, placés sous monitorage en vue d'évaluer les anomalies du segment ST à la phase immédiatement post injection AL. Ils démontrent que l'utilisation d'un $A L$ avec vasoconstricteur ne provoque aucune modification significative su segment ST pris comme indicateur d'une ischémie myocardique. Les recommandations de I'American Dental Association and American Heart Association (1964) précisent que les vasoconstricteurs ne sont pas contre-indiqués dans ces affections lorsqu'une technique anesthésique sûre est utilisée, lorsqu'un test d'aspiration est pratiqué et lorsque la plus petite dose efficace est utilisée.

\subsection{Asthme}

Les vasoconstricteurs associés à une solution anesthésique peuvent être utilisés chez les sujets asthmatique dans le but de maîtriser la douleur et d'éviter le stress qui est probablement la principale source de passage à la crise d'asthme au cabinet dentaire.

L'adrénaline seule est d'ailleurs utilisée pour ses propriétés broncho-dilatatrices dans le traitement de l'asthme et une revue systématique récente rapporte des études cliniques de niveau 3 et 4 qui confirment l'absence d'effets indésirables lors de cette utilisation (Safdar et coll 2001 [NdP IV]). Dans le cas particulier d'asthme cortico-dépendant (Bush et Taylor, 1986 [NdP Ilb]; Perusse et coll 1992, 2 [NdP IV]) le problème de l'hypersensibilité aux sulfites, conservateur du vasoconstricteur, peut se poser. II semble toutefois que $96 \%$ des asthmatiques ne soient pas sensibles au métabisulfite en cause (Send, 1986). Wahl (2000) fait d'ailleurs observer qu'un repas au restaurant contient en moyenne de 25 à $200 \mathrm{mg}$ de sulfites c'est-à-dire 27 fois la dose contenue dans une cartouche de lidocaïne adrénalinée au 1/100 $000(0,9 \mathrm{mg})$.

Le recours à un anesthésique sans vasoconstricteur et sans bisulfite est toutefois indiqué en cas d'asthme cortico-dépendant.

\subsection{Insuffisance hépatique}

Les patients ayant présenté une atteinte hépatique virale ou toxique ancienne et guérie peuvent être traités comme des patients sains. 
En cas d'atteinte sévère évolutive, l'évaluation de la fonction hépatique est importante. La quantité totale injectée peut devoir être réduite et les intervalles entre les injections augmentés sans préjudice de l'utilisation d'un vasoconstricteur associé.

\subsection{Diabète}

Chez les patients diabétiques équilibrés de typel ou II, I'utilisation de vasoconstricteurs est indiquée. En cas de diabète déséquilibré et instable, avec passage brutal de l'hypo à l'hyperglycémie, les quantités d'AL avec vasoconstricteur seront modérées de façon à tenir compte du caractère hyperglycémiant de l'adrénaline (Meechan, 1996 [NdP lb]).

\subsection{Phéochromocytome}

II s'agit d'une tumeur de la médullosurrénale ou du ganglion sympathique para vertébral qui provoque une hypertension sévère en raison de I'hypersécrétion d'adrénaline endogène. En raison du risque de potentialisation des troubles cardiovasculaires, le phéochromocytome et toutes les tumeurs de la médullosurrénale constituent une contre-indication absolue des vasoconstricteurs (Kaufman et coll, 2002 [NdP IV]; Gaudy et Arreto, 1999 [NdP IV]; Perusse et coll, 1992 [NdP IV]). L'injection de solution anesthésique sans vasoconstricteur lorsqu'elle est nécessaire chez le patient atteint d'un phéochromocytome doit avoir lieu en milieu hospitalier et sous monitorage compte tenu des difficultés de stabilisation per-opératoire de la pression artérielle chez ces patients (Niruthisard et coll, 2002 [NdP III]; Tanaka et coll, 1991 [NdP III]; Pratilas et Pratila, 1979 [NdP IV]).

\subsection{Os irradié}

Toute irradiation des structures maxillo-faciales à visée thérapeutique que ce soit sous forme de brachy ou de téléradiothérapie, réduit la vascularisation de l'os de sorte que le tissu osseux n'est plus capable de se défendre contre les agressions. Un œdème suivi d'une nécrose endothéliale entraîne successivement la hyalinisation, la fibrose et la thrombose au sein de la paroi des vaisseaux irradiés. Les vaisseaux s'oblitèrent et l'hypoxie tissulaire aboutit à la lyse du collagène puis à une dégénérescence de la médullaire osseuse (Marx, 1983 [NdP III]). C'est l'ostéoradionécrose (ORN) ou ostéite radique qui constitue I'une des complications majeures des irradiations thérapeutiques maxillo-faciales.

On comprend aisément qu'un tel processus qui se produit généralement pour des irradiations supérieures à $60 \mathrm{~Gy}$ puisse être favorisé par l'ischémie locale provoquée au point d'injection d'un AL additionné de vasoconstricteur.

Chez l'animal, Heiss et Grasser (1968) [NdP Ib] ont démontré dans des conditions expérimentales extrêmes l'augmentation significative du risque d'ORN après injection de substances vasoconstrictrices au contact de mandibules de rats irradiées.

II n'existe évidemment pas chez l'homme de protocole comparable. En revanche, Maximiw et coll 1991 [NdP IIb] ont démontré que l'utilisation de faibles doses de vasoconstricteurs ou de solutions AL sans vasoconstricteur aboutissaient, pour un groupe de 449 avulsions effectuées dans un os ayant reçu en moyenne $50 \mathrm{~Gy}$ (extrêmes à 25 et $84 \mathrm{~Gy}$ ), à une absence totale d'ostéoradionécrose post extractionnelle et ce après un suivi post-extractionnel de 4,8 ans en moyenne.

Bien que les facteurs de risque clairement identifiés des ORN post extractionnelles soient le site mandibulaire, la dose totale délivrée et le mode d'irradiation (Curi et Dib, 1997 [NdP III]) et qu'il n'existe aucune évaluation chez I'homme du risque direct lié aux vasoconstricteurs; il paraît souhaitable d'éviter l'association de vasoconstricteurs à l'AL lors des soins conservateurs et surtout non conservateurs sur un os irradié au delà de $\mathbf{4 0} \mathbf{~ G y}$.

\section{Etats physiologiques et vasocons- tricteurs}

\subsection{Grossesse et lactation}

Bien que les vasoconstricteurs (surtout la noradrénaline) disposent d'un potentiel de réduction de la perfusion placentaire, les études menées médecine buccale chirurgie buccale VOL. $9, \mathrm{~N}^{\circ}$ 2003 page 87 
médecine buccale chirurgie buccale

)L. $9, \mathrm{~N}^{\circ} 2$

2003

page 88 sur ce sujet n'ont démontré aucun effet indésirable sur le fœtus pour l'adrénaline (Haas et coll, 2000 [NdP III]). En réalité, les doses d'adrénaline utilisées dans les solutions anesthésiques locales commercialisées sont si faibles qu'il est très improbable qu'elles puissent affecter le débit sanguin utérin. S'agissant de la lactation les seules données disponibles sont des opinions d'auteurs. Elles confirment la possibilité d'utiliser les vasoconstricteurs en association avec les AL chez la femme au cours de l'allaitement (Gibbs et Hawkins, 1994 [NdP IV]; Malamed, 1997 [NdP IV).

\subsection{L'enfant}

Chez l'enfant, la prise en charge de la douleur se fait classiquement en évitant un recours systématique aux vasoconstricteurs. Cette pratique est sans rapport avec le risque toxique. Elle découle de l'augmentation du risque de morsure sévère de la région labiale anesthésiée longtemps après la fin des soins en raison de l'allongement de la durée de l'anesthésie en présence d'un vasoconstricteur (Walh, 1997 [NdP IV]; Gaudy et Aretto, 1999 [NdP IV]). Cela concerne principalement l'anesthésie loco-régionale du nerf mandibulaire et le danger de morsure de la lèvre inférieure.

Hersh et Hermann (1995) [NdP Ib] ont néanmoins montré qu'il n'y avait pas de différence significative dans la durée de l'anesthésie labiomentonnière après injection de mépivacaïne sans vasoconstricteur lorsqu'on la compare avec une injection de lidocaïne+adrénaline. Selon ces auteurs, le recours à un anesthésique sans vasoconstricteur chez l'enfant est donc sans intérêt et ils recommandent au contraire l'utilisation de la lidocaïne $2 \%$ adrénalinée jusqu'à une dose totale de 4,4 carpules de 1,8 ml chez un enfant de $25 \mathrm{~kg}$ contre 2,8 cartouches de mépivacaine $3 \%$ sans adrénaline.

Notons que Hersh et coll (1991) [Non classé : cas clinique] ont rapporté un surdosage mortel chez une enfant de 5 ans pesant 16,4 kg et chez laquelle on avait injecté 5 cartouches de 1,8 ml de mépivacaine $3 \%$ sans vasoconstricteur.
Il existe différentes méthodes complexes de calcul de la dose chez l'enfant en fonction de la dose d'anesthésique chez l'adulte, et en fonction de la surface corporelle rapportée au poids. Les formules différentes de Clark et Young aboutissent à des résultats voisins. L'ADA et la FDA proposent les tables de conversion figurant dans les deux tableaux 1 et 2 .

Bien qu'en pratique le problème se pose rarement, l'utilisation des anesthésiques locaux et du vasoconstricteur chez l'enfant de moins de 6 mois est contre-indiquée compte tenu des faibles capacités métaboliques qui peuvent conduire à un surdosage ou à une accumulation de la fraction libre.

Tableau 1 : Dose enfant en fonction du poids calculée en fonction de la dose habituelle de l'adulte. D’après Berini et Gay, 1997.

\begin{tabular}{|cc|}
\hline $\begin{array}{c}\text { Poids de l'enfant } \\
\text { en } \mathbf{~ k g}\end{array}$ & $\begin{array}{c}\text { Fraction de la } \\
\text { dose adulte }\end{array}$ \\
\hline 10 & 0,27 \\
15 & 0,36 \\
20 & 0,48 \\
25 & 0,55 \\
30 & 0,62 \\
35 & 0,69 \\
40 & 0,75 \\
\hline
\end{tabular}

Tableau 2 : Comparaison des doses totales recommandées par la Food and Drug Administration en lidocaïne à $2 \%$ adrénalinée et Mépivacaïne chez l'adulte et l'enfant. D'après Walh, 1997.

\begin{tabular}{|c|c|c|}
\hline & $\begin{array}{c}\text { Mépivaca } \\
\text { à } 3 \%\end{array}$ & $\begin{array}{c}\text { Lidocaïne } 2 \% \\
\text { tadrénaline }\end{array}$ \\
\hline $\begin{array}{l}\text { Quantité d'anesthésique } \\
\text { par carpule de } 1.8 \mathrm{ml}\end{array}$ & $54 \mathrm{mg}$ & $36 \mathrm{mg}$ \\
\hline $\begin{array}{l}\text { Nombre maximal de } \\
\text { cartouches par } 24 \text { heures } \\
\text { pour un adulte de } 70 \mathrm{~kg}\end{array}$ & 7,4 & 13,9 \\
\hline $\begin{array}{l}\text { Nombre maximal de } \\
\text { cartouches par } 24 \text { heures } \\
\text { pour un enfant de } 25 \mathrm{~kg}\end{array}$ & $S$ & 4,4 \\
\hline
\end{tabular}




\subsection{Le sujet âgé}

Le sujet âgé est souvent la cible des différentes affections qui ont déjà été passées en revue dans ce rapport. II est en outre classique de considérer les sujets au delà de 70 ans comme des insuffisants rénaux chroniques (après

\section{RÉFÉRENCES}

1 - Ackerman B, Helberg IB, Trossvik C. Primary evaluation of the local anesthetic properties of the aminoamide agent ropivacaine. Acta Anaesthesiol Scand 1988; 32: 571-8.

2 - Allwood MJ, Cobbold AF, Ginsburg J. Peripheral vascular effects of nor adrenaline, isopropyl nor adrenaline and dopamine. Br Led Bull 1963; 19: 132-8.

3 - American Dental Association and American Heart Association. Management of dental problems in patients with cardiovascular disease: report of a working conference jointly sponsored by the American Dental Association and American Heart Association. J Am Dent Assoc 1964; 68: 333-42.

4 - Anguera Camos I, Brugada Terradellas P. Nuevas Perpspectivas en el tratamiento no farmacologico de la fibrilacion auricular. Med Clin 2000; 114: 25-30.

5 - BerinI L, GaY C. Anestesia Odontologica. Avances Editeur, Madrid 1997.

6 - BeRLING C. Carbocain in local anesthesia in the oral cavity. Odont Rev y 1958, 9: 254-8.

7 - Besner E. Systemic effects of racemic epinephrine when applied to the bone cavity during periapical surgery. Va Dent J 1972; 49: 9-12.

8 - Blinder D, Shemesh J, TAICher S. Electrocardiographic changes in cardiac patients undergoing dental extractions under local anaesthesia. J Oral Maxillofac Surg 1996; 54: 162-5.

9 - BOAKES AJ . Adverse reactions to local anesthetic vasoconstrictors preparations. Br Dent J. 1972; 1: 133-7.

10 - BOAKES AJ . Interactions between sympathomimetic amines and antidepressant agents in man. $\mathrm{Br}$ Dent J 1973; 1: 311-4.

11- BonICA JJ. Labour pain in Textbook of pain (pp624-6). Churchill Livingstone Editeurs, 3 e edition, 1999.

12 - Borea G, Montebugnoli L, Capuzzi P, Vaccaro MA. Ciruclatory dynamics during dental operations in patients with heart transplants. Quintessence Int1993; 24: 749-51.

13 - Brand HS, Van der Wal J HA, Palmer Bouva CCR, DE VRIES DR. Cardiovascular changes during subgingival debridement. Int Dent J 1997; 47 : 110-4. 40ans la filtration glomérulaire baisse de $1 \mathrm{ml}$ par minute et par an) ce qui impose de diminuer les doses totales d'un tiers de 70 à 80 ans et de la moitié au delà (Commissionnat et Rimet, 1992 [NdP IV]).
14 - Brand hS, Abraham-InpIjn L. Cardiovascular responses induced by dental treatment. Eur J Oral Science 1996; 104: 245-52.

15 - Braun $H$. Über dein Einfluss der Vitalitat der Gewebe auf die Örtlichen und allgemeinen giftwirkjung lokaler anästhesierender mitteln und über bedeutjung des Adrenalins für die lokalanesthasie. Arch Klin Ckr 1903; 69: 541-91.

16- Braun H. Local Anesthesia Lea et Febinger Publishers, New York 1924.

17 - Britz-Mckibin P, Kranack AR, Paprica A, Chen DDY. Quantitative assay of epinephrine in dental anesthetic solutions by capillary electrophoresis. Analyst 1998; 123: 1461-3.

18 - Britz-MckibiIn P, Wong J, Chen DDY. Analysis of epinephrine from fifteen different dental anesthetic formulations by capillary electrophoresis. J Chromat 1999; 853: 535-40.

19 - Bromage PR, C amporesi EM, Durant PA. Influence of epinephrine as an adjuvant to epidural morphine. Anesthesiol 1983; 58: 257-61.

20 - Bush RK, TAYLOR SL. Prevalence of sensitivity to sut fiting agents in asthmatic patients. Am J Med 1986; 74: 687-91.

21 - BUDENTZ A. Local anaesthetics and medically complex patients. J Calif Dent Ass 2000; 28: 611-19.

22 - Camara AK, Turner LA, Bosnj AK ZJ . Interactions of halothane with isoproterenol and epinephrine on canine epicardial conduction velocity at normal and elevated potassium levels. Acta Anaesth Scand 2001; 45: 885-92.

23 - Campbell J H, Huizinga PJ, Das SK, Rodriguez J P, GOBETTI J P. Incidence and significance of cardiac arrhythmia in geriatric oral surgery patients. Oral Surg Oral Med Oral Pathol Oral Radiol Endod 1996; 82: $42-6$.

24 - CANNELL H. Evidence of safety margins of lignocaine local anesthetics for peri-oral use Br Dent J 1996; 181: 243-9.

25 - Cantaloube D, Rives J M, Daize E, Salleras J P. Traitement préventif et curatif des hémorragies opératoires. Encycl Med Chir Editions techniques Editeur, Paris 22090G 10, 1991. médecine buccale chirurgie buccale VOL. $9, \mathrm{~N}^{\circ}$ 2003 page 89 
26 - Cawson RA, Curson I, WhitTington DE. The hazards of dental local anaesthetics. Br Dent J 1983; 154: 253-8.

27 - Chamberlain TM, Davis RD, Murchinson DF, Hansen SR, RICHARDSON BW. Systemic effects of an intra osseous injection of $2 \%$ lidocaïne with $1 / 100000$ epinephrine. Gen Dent 2000; 48: 299-302.

28 - Chen J, Dohi S, Tan Z, Banno Y, Nozawa Y. The inhibitory effect of local anesthetic $s$ on bradykinin-induced phospholipase $D$ activation in rat pheochromocytoma PC12 cells. Anesth Analg 2002; 95: 88-97.

29 - Cheraskin MD, Prasertsuntarasal MS. Use of epinephrine with local anaesthesia in hypertensive patients. Effects of sedation on blood pressure and pulse rate in the waiting room. J Am Dent Assoc 1958; 56: 210-8.

30 - Cheraskin MD, Prasertsuntarasai MS. Use of epinephrine with local anaesthesia in hypertensive patients. Effects of tooth extraction on blood pressure and pulse rate. J Am Dent Assoc 1959; 58: 61-8.

31- Chernow B, Balestrieri F, Ferguson CD, Terezhalmy GT, Fletcher J R, LaKe CR. Local dental anaesthesia with epinephrine. Minimal effects on the médecine buccale chirurgie buccale

L. $9, N^{\circ} 2$

2003

page 90 sympathetic nervous system or on hemodynamic variables. Arch Intern Med 1983; 143: 2141-3.

32 - ChIU YC, BReCht K, DAS Gupta DS, MhoON E. Myocardial infarction with topical cocaine anaesthesia for nasal surgery. Arch Otolaryngol Head Neck Surg 1986; 112: 988-90.

33 - Christensen LQ, Bonde J , Kampmann J P. Drug interactions with inhalational anaesthetics. Acta Anaesth Scand 1993; 37: 231-44.

34 - Cioffi GA, Chernow B, Glahn RP, Terezhalmy GT, LAKE CR. The hemodynamic and plasma cathecolamine responses to routine restorative dental care. J Am Dent Assoc 1985; 111: 67-70.

35 - Coggins R, Reader A, Nits Beck M, Meyers W. Anaesthetic efficacy of intraosseous injection in maxillary and mandibular teeth. Oral Surg Oral Med Oral Pathol Oral Radiol Endod 1996; 81: 634-41.

36 - Commissionat Y, Rimet J L. Anesthésie Locale. Complications. Actual Odontostomatol 1992; 179: 497-506.

37-COVINO BG, VASSALLO HG. Local anesthetics: mechanism of action and clinical use. Grune and Stratton Editeur, New York 1976.

38 - CURI MM, DIB LL. Osteoradionecrosis of the jaws: a retrospective study of the background factors and treatment in 104 cases. J Oral Maxillofac Surg 1997; 55: 545-6.

39 - Dagher FD, Ghassan MY, Machtou P. An evaluation of $2 \%$ lidocaine with different concentrations of epinephrine for inferior alveolar nerve block. J Endod 1997; 23: 178-80.

40 - DAMm DD, FANTASIA J E. Recurring palatal ulceration Gen Dent 1992; 40: 102 et 148

41 - Daublander M, ROth W, Lipp M. Effects of different lidocaïne sprays for topical anesthesia on cardio- vascular parameters and oxygen saturation. Anesth Pain Cont Dent 1992; 1: 161-6.

42 - Daublander M, Muller R, Lipp MDW. The incidence of complication associated with local anaesthesia in dentistry. Anesth Prog 1997; 44: 132-41.

43 - DaVIES AP, LefKowitz RJ . Regulation of adrenergic receptors in Lefkowitz Receptors regulation. Chapmann and Hall Editor, New York 1981.

44 - Deanfield Je, Ribierov P, Oakley K, Krikler $S$ SELWYN AP. Analysis of ST segment changes in normal subjects: implication for ambulatory monitoring in angina pectoris. Am J Cardiol 1984; 54: 1321-5.

45 - DI ANGelis N, LUEPKER RC. The effect of the dental setting on blood pressure measurement. Am J Public Health 1983; 73: 1210-2.

46 - Dionne RA, Goldstein DS, Wirdzek PR. Effects of diazepam premedication and epinephrine-containing local anaesthetic on cardiovascular and plasma catecholamine responses to oral surgery. Anesth Analg 1984; 63: 640-6.

47 - Dunbar D, Reader A, Nist R, Beck M, Meyers W. Anaesthetic efficacy of intraosseous injection after an inferior alveolar nerve block. J Endod 1996; 22: 481-6.

48 - Engquist A, Brandt MR, Fernandes A, Kehlet H. The blocking effects of epidural analgesia on the adrenocortical and hyperglycaemic responses to surgery. Acta Anaesth Scand 1977; 21: 330-5.

49 - EzZat Abouleish MB, Klionsky BL, TaYLOR FL. Lidocaine effects on leucocytes and erythrocytes. Anesthesiology 1978; 48: 295.

50 - Falaiye VO, RoOd J P. Influence of adrenaline on the onset time of lignocaine anaesthesia. J Dent 1990; 18: 221-3.

51 - Fink BR, AAsheim GM, LeVy BA. Neural pharmacokinetics of epinephrine. Anesthesiology 1978; 48: 263-8.

52 - Foitzik C, Daublander M, Vietor K, Protsch von ZIETEN A. Management and care of patients at risk in a surgical dental practice. Anesth Prog 1996; 43: 73-7.

53 - Foster CA, Aston S] . Propanolol-epinephrine interaction: a potential disaster. Plast Reconstr Surg 1983; 72: 74-8.

54 - Galili D, Kaufman E, Garfunkel AA, Michaeli Y. Intraligamentari anaesthesia: a histological study. Int J Oral Surg 1984; 13: 511-6.

55 - GANGAROSA LP, HaLIK FJ . A clinical evaluation of local anaesthetic solutions containing graded epinephrin concentrations. Arch Oral Biol 1967; 12: 611-21.

56 - GAUdy J F, ARreto CD. Manuel d'analgésie en odontostomatologie. Masson Editeur, Paris 1999.

57 - Germishuys PJ . Hyperresponders and adrenaline in local anaesthetic solutions. South Afr Dent J 2001; 56: 175-7.

58 - GiBBS CP, Hawkins J L. Anesthesia for the pregnant patient requiring no obstetrical surgery. ASA Refresher course in anaesthesiology 1994; 22: 127-40. 
59 - Goldman V, Astrom A, Evers $H$. The effect of tricyclic antidepressants on the cardiovascular effects of local anaesthetic solutions containing different vasoconstrictors. Anaesthesia 1971; 26: 91-6.

60 - Goldstein DS, Dionne R, Sweet J, Gracely R, Brewer $B, G$ regg R, KeISER HR. Ciruclatory plasma catecholamine lipid and psychological responses to real-life stress (third molar extraction): effects of diazepam sedation and of inclusion of epinephrine with the local amesthetic. Psychosomatic Med 1982 44: 259-72.

61 - Gortzak Rat, Abraham Inpij n L, Peters G. Non invasive 27 hour blood pressure registration including dental checkups in some dental practice. Clin Prevent Dent 1992; 14: 5-10.

62 - Gortzak RAT, Abraham InPIJ n L, Oosting J. Blood pressure response to dental checkup: a continuous, non-invasive registration. Gen Dent 1991; 39: $339-42$

63 - Gortzak Rat, Abraham InPin n L, Peters G. Non invasive 27 hour blood pressure registration including dental checkups in some dental practice. Clin Prevent Dent 1992; 14: 5-10.

64 - Gray RJ , Lomax AM, Rood J P. Periodontal ligament injection: alternative solutions. Anesth Prog 1990; 37: 293-5.

65 - Grossman LI. Endodontic practice Lea et Febiger editor, 7e ed, Philadelphia 1970.

66 - Guglielmo A, Reader A, Nist R, Beck M, Weaver J . Anesthetic efficacy and heart rate effect of the supplemental intraosseous injection of $2 \%$ mepicacaine with $1 / 20000$ levonordefrin. Oral surgery Oral Med Oral Pathol 1999; 87: 284-93.

67- GUTMAN J L. Parameters in achieving quality anaesthesia and hemostasis in surgical endodontics. Anesth Pain Control Dent 1993, 223-6.

68 - GutMAn J L, HaRRISON JW. Surgical endodontics, Ishiyaku Euro America Editor, St Louis 1994.

69 - HAAS DA, LENNON D. A 21 year retrospective study of reports of paresthesia following local anaesthetic administration J Can Dent Assoc 1995; 4: 319-30.

70 - HAAS DA, PYNn BR, SANDS TD. Drug use for the pregnant or lactating patient. Gen Dent 2000; 48: 5460.

71 - HandLeR LE, ALBERS DD. The effect of the vasoconstrictor epinephrine on the duration of pulpal anesthesia using the intraligamentary injection J Am Dent Assoc 1987; 114: 807-9.

72 - Hansbrough Jf, Near A. Propanolol -epinephrine antagonism with hypertension and stroke. Ann Int Med 1980; 92: 717-21.

73 - HANSTEN PD. Drug interactions affecting the cardiovascular response to sympathomimetics. Drug Interac Newsletter 1981; 1: 21-6.

74 - Hayachi Y, Kamibayashi T, SUmikawa K, Yamatodani A, Kuro M, Yoshiya I. Adrenoreceptor mechanism involved in thiopental-epinephrine induced arrhythmias in dogs. Am J Physiol 1993; 265: 1380-5.
75 - HeISS J. Grasser H. Behaviour of the rat mandible following maximum irradiation with simultaneous administration of vaso-active and vasoconstricting substances. Dtsch Zahnarztlz 1968; 23: 1410-7.

76 - HeRsh EV, HeLPIN ML, Evans OB. Local anesthetic mortality: report of a case. J Dent Child 1991; 58: 489-91.

77 - HeRSh EV, Hermann DG. Assessing the duration of mandibular soft tissue anaesthesia J Am Dent Assoc 1995; 126: 1531-6.

78 - Hilley MD, Miliam SB, Gieske AH, Giovannitti J A. Fatality associated with the combined use of halothane and gingival retraction cord. Anesthesiology 1984; 60: 587-8.

79- Himuro H, Murata T, Tamesue A, Nakajima T, Hiratsuka M, Tsubaki T, Aono K. Determination of the desirable epinephrine concentration containing in dental local anaesthetic solution. Comparison between two lidocaine solutions containing1/80000 and 1/200000 epinephrine. Fukuoka Shika Daigaku Gakkai Zasshi 1989; 16: 323-7.

80- HONDRUM SO. Hypertensive episode in the dental office. Gen Dent 1985; 33: 134-9.

81- Hosoda R, HatTori M, Shimada M, Shimomura $Y$, MatsuURA $M$. Favourable effects of epidural analgesia on hemodynamics, oxygenation, and metabolic variables in the immediate post anaesthesic period. J J ap Soc Clin Anesth 1991; 11: 570-9.

82 - Houston J B, Appleby R, De Counter L, Callaghan N, Funk DC. Effect of repinephrine-impregnated retraction cord on the cardiovascular system. J Prosth Dent 1970; 24: 373-6.

83- ICHINOHE T, FukUdA K, Kaneko Y. Epinephrine at doses used in dentistry deteriorates platelet retention rate. Anesth Prog 1997; 44: 59-63.

84 - INGLE J L. Endodontics, Lea et Febiger éditeur, Philadelphie 1965.

85 - J Acquot C, Rapin JR, Vielllefosse R. Pharmacologie dentaire pratique, Julien Prélat Editeur, Paris 1978.

86 - J AGE J. Circulatory effects of vasoconstrictors combined with local anaesthetics. Anesth Pain Cont Dent 1993; 2: 81-6.

87 - J OHNSON WC, WIDRICH WC. Control of bleeding varices by vasopressine: a prospective randomised study. Ann Surg 1977; 186: 369-75.

88 - J ORKJ END L, S KOGLUND LA. A doses response study of adrenaline with lignocaine $2 \%$ : effect on acute postoperative pain after oral soft tissue surgery. $\mathrm{Br}$ J Clin Pharmacol 2000; 51: 335-41.

89 - J ORKJ ENd L, SKoglund LA. Increase in volume of lignocaine/adrenaline containing local anaesthetic solution causes increase in acute postoperative pain after gingivectomy. Br J Oral Maxillofac Surg 2000; 38: $230-4$

90 - Kabambe WM, Rood J P, Sowray J H. A comparison of plain 2 per cent lignocaine and 2 per cent lignocaine with adrenaline in local analgesia for minor oral surgery. J Dent 1982; 10: 136-9. médecine buccale chirurgie buccale VOL. $9, \mathrm{~N}^{\circ}$ 2003 page 91 
91 - Kaufman E, Garfunkel A, Findler M, Elad S, Zusman $S P$, Malamed SF, Galili D. Emergencies evolving from local anesthesia. Refuat Hapeh Vehashinayim 2002; 19: 13-8.

92 - KeESKLING GR, HINDS EC. Optimal concentration of epinephrine in lidocainne solutions. J Am Dent Assoc 1963; 66: 337-40.

93 - Kennedy M, Reader A, Beck M, Weaver J . Anesthetic efficacy of ropivacaine in maxillary anterior infiltration. Oral Surg Oral Med Oral Pathol 2001; 91: 406-12.

94 - Khoury F, Hinterthan A, Schurman J, Arns H. Clinical comparative study of local anesthetics. Random double blind study with four commercial preparations. Dtsch Zahnarztl 1991; 46: 822-4.

95 - Kleemann PP, Lanz E, Schmitt B, Von Domarus H. Ist das Risiko einer Lokalinjektion von ornipressin wohnend der Vollnarkose vertretbar? Dtsch Z Mund Kiefer Gesichtschir 1986; 10: 14-8.

96 - KNOLL-KOHLER E. Sicherheit bei der lokalanasthesiell. Pharmakologie vasokonstriktorischer. Zusatze. Phillip J 1988; 1: 79-87.

97-KNOLL-Kohler E， Brandt K， Becker J. Cardiohemodynamic and serum catecholamine response to surgical removal of impacted mandibular third molars under local anaesthesia: a randomised double blind parallel group and crossover study. J Oral and Maxillofacial surg 1991; 49: 957-62.

98 - KnOlL-Kohler E, Fortsch G. Pulpal anaesthesia dependent on epinephrine dose in $2 \%$ lidocaïne. Oral Surg Oral Med Oral Pathol 1992; 73: 537-40.

99 - Knoll Kohler E, Brandt K, Knoller M. The beta mimetic effects following the endoral injection of epinephrine with articaine. Schweiz Monatsschr Zahnmed 1992; 102: 172-7.

100 - Lathers CM, Tyau LS, Spino MM, Agarwal I. Cocaine induced seizures, arrhythmias and sudden death. J Clin Pharmacol 1988; 28: 584-93.

101 - Lipp M, Dick W, Daublander M, Fuder H, S tantonHICKS M. Exogenous and Endogenous plasma levels of epinephrine during dental treatment under local anaesthesia. Reg Anesthesia 1993; 18: 6-12.

102 - Mac Lean ME, Wayman BE, Mayhew RB. Duration of anesthesia using the periodontal ligament injection: a comparison of bupivacaine to lidocaïne. Anesth Pain Cont Dent 1992; 4: 207-13.

103 - Mac Lean C, Reader A, Beck M, Meryers WJ. An evaluation of 4 ù prilocaine and $3 \%$ mepivacaine compared with $2 \%$ lidocaïne (1/100 000 epinephrine) for inferior alveolar nerve block. J Endod 1993; 19: 146-50.

104 - Madrid C, Caballero R, Duran D, Bru de Sala C. L'anesthésie Mandibulaire. Réal C lin 1991; 2: 51-68.

105 - MaLAmed S. Electrocardiographic changes in cardiac patients undergoing dental extractions under local anesthesia Discussion. J Oral Maxillofacial Surg 1996; 54: 165-6.

106 - MaLAmed SF. Handbook of local anesthesia. Mosby Editor, 4e Edition, St Louis 1997.
107 - Mamiya H, IChinohe T, Kaneko Y. Effects of block analgesia on attenuating intraoperative stress response during oral surgery. Anesth Prog 1997; 44: 101-5.

108 - Marx RE. Osteoradionecrosis a new concept of its pathophysiology. J Oral Maxillofac Surg 1983; 41: 283-8.

109 - Massalha R, Valdman Farkash P, Merkin L. Fatal intracerebral haemorrhage during dental treatment Int J Med Sci 1996; 32: 774-6.

110 - MaXIMYW WG, WoOd RE, LIU FF. Post radiation dental extractions without hyperbaric oxygen. Oral Surg Oral Med Oral Pathol Oral Radiol Endod 1992; 74: 155-7.

111 - Meechan J G. Epinephrine Magnesium and dental local anaesthetic solutions. Anesth Prog 1996; 43: 99-102.

112 - Meechan J G. Plasma potassium changes in hypertensive patients undergoing oral surgery with local anaesthetic containing epinephrine. Anesth Prog 1997; 44: 106-9.

113 - Meechan J G, Skelly AM. Problems complicating dental treatment with local anaesthesia or sedation: prevention and management. Dental Update 1997; 24: 278-83.

114 - Meechan J G, Rood J P. Adverse effects of dental local anaesthesia. Anaesthesia, 1997; 24: 315-8.

115 - Meechan J. How to avoid local anaesthetic toxicity. BrDent J 1998; 184: 334-5.

116 - Meechan J G, Day PF, Mc Millan AS. Local anesthesia in the palate: a comparison of techniques and solutions. Anesth Prog 2000; 47: 139-42.

117 - Meechan J G, Cole B, Welbury RR. The influence of two different dental local anaesthetic solution on the haemodynamic responses of children undergoing restorative dentistry: a randomised, single-blind, split-mout study. Br Dent J 2001; 190: 502-4.

118 - MeYer FU. Haemodynamic changes under emotional stress following a minor surgical procedure under local anaesthesia. Int J Oral Maxillofac Surg 1987; 16: 688-94.

119 - Mito RS, YAGIELA J A. Hypertensive response to levonordefrin in a patient receiving propanolol : report of a case. J Am Dent Assoc 1988; 116: 55-7.

120 - Montebugnoli L, Pelliccioni GA, Borghi C. Effetti del vasocostricttore durante l'aestesia locale. Dental Cadmos 1990; 58: 62-71.

121 - Moore PA, Gage TW, Hersh EV, Yagiela J A, haAS DA. Adverse drug interaction in dental practice. J Am Dent Assoc 1997; 130: 47-54.

122 - Moskowitz MA. The neurobiology of vascular head pain. Ann Neurol 1984; 16: 157-68.

123 - Moyer J A, Greenberg LH, Frazer A, Brunswick DJ, Mendels J, Weiss B. Opposite effects of acute and repeated administration of desmethylimipramine on adrenergic responsiveness in rat pineal gland. Life Sci 1979; 24: 2237-44.

124 - MuZYKA B. Atrial fibrillation its relationship to dental care. J Am Dent Assoc 1999; 130: 1080-5. 
125 - MyeRs RR, HeckMAnn HM.Effects of local anaesthetics on nerve blood flow using lidocaine with and without epinephrine. Anesthesiology 1989; 71: 757-62.

126 - NaKaI Y, M ILGRom P, M ANCL L, COLdWELL S, Domoto PK, RamsaY DS. Effectivemess of local anesthesia in pediatric dental practice. J Am Dent Assoc 2000; 131: 1699-705.

127 - NiRuthisard S, Chatrkaw P, LaORnual S, Sunthornyothin S, Prasertsri S. Anesthesia for one-stage bilateral pheochromocytoma resection in a patient with MEN type lla : attenuation of hypertensive crisis by magnesium sulfate. J Med Assoc Thai 2002; 85: 125-30.

128 - Niwa H, ShiButani T, HORI T, KIM Y, AKITA M, MatsuURa $H$. The interaction between pindolol and epinephrine contained in local anesthetic solution to the left ventricular diastolic filling velocity in normal subjects. Anesth Prog 1996; 43: 78-84.

129 - Niwa H, SATOH Y, MatsuURa H. Cardiovascular response to epinephrine containing local anaesthetics for dental use: a comparison of haemodynamic responses to infiltration anesthesia and ergometer stress testing. Oral Surg Oral Med Oral Pathol Oral Radiol Endod 2000; 90: 171-81.

130 - OdOR TM, P ITT FORD TR, MC DONALd F. Andrenaline in local anaesthesia: the effect of concentration on pulpal circulation and anaesthesia. Endod Dent Traumatol 1994; $10: 167-73$.

131 - Oertel R, Rahn R, KIRCH W. Clinical pharmacokinetics of articaine. Clin Pharmacokin 1997; 33: 417 25.

132 - OKADA Y, SUZUKI H, ISHIYAMA I. Fatal subarachnoid haemorrhage associated with dental local anesthesia. Australian Dental J 1989; 34: 323-5.

133 - PallasCh TJ . Vasoconstrictors and the heart. J Calif Dent Assoc 1998; 26: 668-73.

134 - Palmer Bouva C, Oosting J, de Vries R, AbrahamINPIJ N L. Stress in elective dental treatment : epinephrine, norepinephrine, the VAS, ansd CDAS in four different procedure. Gen Dent 1998; 46: 356-60.

135 - PeRSSON G, Simers B. The risk of potentialting effect of local anesthesia with adrenaline in patients treated with tricyclic antidepressants. Sven Tandlak Tidskr 1975; 68: 9-18.

136 - Perusse R, Goulet J P, Tutcotte J Y. Contraindications to vasoconstrictors in dentistry Part I: cardiovascular diseases. Oral Surg Oral Med Oral Pathol 1992; 74: 679-86.

137 - Perusse R, Goulet JP, Tutcotte J Y. Contraindications to vasoconstrictors in dentistry Part II: hyperthyroidism, diabetes, sulphite sensitivity, cortico-dependent asthma and pheochromocytoma. Oral Surg Oral Med Oral Pathol 1992; 74: 687-91.

138 - PetrikAs AZ. A clinico-pharmacologic study of intraseptal spongiosa dental anesthesia with lidocaine and with/without adrenaline. Stomatologia 1990; 69: 27-9.
139 - Pratilas V, Pratilas MG. Anaesthetic management of phaeochromocytoma. Can Anaesth Soc J 1979; 26: 253-9.

140 - Quyrumi AA, Mockus L, Wright C, Fox KM. Morphology of ST segment changes in patients with varying severity of coronary artery disease: investigation of the frequency of nocturnal ischaemia and coronary spasm. Br Heart J 1985; 53: 186-93.

141 - Reitz J, Reader A, Nist R, Beck Meyers WJ. Anesthetic efficacy of the intraosseous injection of $0.9 \mathrm{ml}$ of $2 \%$ lidocaine (1:100 000 epinephrine) to augment an inferior alveolar nerve block. Oral Surg Pral Pathol Oral Med 1998; 86: 516-23.

142 - Replogue K, Reader A, Nist R, Beck M, Meyers W. Cardiovascular effects of the intraosseous injection of $2 \%$ lidocaïne with 1:100 000 epinephrine and $3 \%$ mepivacaine. J Am Dent Assoc 1999; 130: 649-57.

143 - ROBERTS DH, SOWRAY J H. Local analgesia in dentistry. Wright éditeur, 3e édition, B ristol 1987.

144 - Rood J P. Local Anesthesia dosage. Br Dent J 1998; 185: 59.

145 - Rood J P. Safety of adrenaline. Br Dent J 1999; 186: 104.

146 - Ruffolo RR, Vichols AJ, Stadel J M, Hieble J P. Structure and function of $\alpha$ adrenoreceptors. Pharmacol Rev 1991; 43: 475-505.

147 - SACK U, KLEEMAN PP. Intraoral conduction anesthesia with epinephrine containing local anaesthetics and arterial epinephrine plasma concentration. Anesth Pain Cont Dent1992; 1: 77-80.

148 - Safdar B, Cone DC, Pham KT. Subcutaneous epinephrine in the prehospital setting. Prehosp Emergency Care 2001; 5: 200-7.

149 - Sakyray M. Effect of epinephrine in local dental anesthetics on plasma catecholamine concentration and circulation. Dentistry in J apan 1990; 27: 135-9.

150 - Santoro V. Marsicano C. Variazione dei parametri emodinamici in pazienti in anestesia generale sottoposti ad infiltrazione sottomucosa con miscela di anestesico locale/adrenalina. Minerva Stomatologica, 1998; 47: 649-53.

151 - SenG GF, GAY BJ. Dangers of sulphites in dental local anaesthetic solutions: warning and recommendation. J Am Dent Assoc 1986; 113: 769-70.

152 - Shanks CA. Intravenous octapressin during halothane anaesthesia: a pilot study. Br J Anaesth 1963; 35: 640-7.

153 - Silviestre FJ, Verdu MJ, Sanchis J M, Grau D. Penarrocha M. Efectos de los vasoconstrictors usados en odontologia sobre la presion arterial sistolica y diastoloca. Med Oral 2001; 6: 57-63.

154 - Sommer R, Ostrander F Crowley M. Clinical Endodontics, Saunders Editor, Philadelphia, 1962

155 - Sunada K, Nakamura K, Yashimoro M, Sumitomo M, FURUYA $H$. Clinically safe dosage of felypressin for patients with essential hypertension. Anesth Prog 1996; 43: 108-15. médecine buccale chirurgie buccale VOL. $9, \mathrm{~N}^{\circ}$ 2003 page 93 
médecine buccale chirurgie buccale
156 - SYNGCUK K, S IVAKAmI R. Haemostasis in endodontic surgery. Dent Clin North Amer 1997; 41: 499-511.

157 - Tagger E, TAgger M, Sarnat H, Mass E. Periodontal ligament injection in the dog primary dentition: spread of local anaesthetic solution. Int J Paediatr Dent 1994; 4: 159-66.

158 - Tanaka S, Miyabe M, Ohyama I, Seki S, Tsukamoto T, NAMIKI A. Sevoflurane with continuous epidura anesthesia for removal of pheochromocytoma. Masui 1991; 40: 1261-4.

159 - Tolas AG, Pflug aE, Halter J B. Arterial plasma epinephrine concentrations and haemodynamic responses after dental injection of local anaesthetic with epinephrine. J Am Dent Ass 1982; 104: 41-3.

160 - TORdofF SG, BROSSY M, ROWBOTHAM DJ , J AMES J N, J AMES R, RAPHAEL J H. The effect of preincisional infiltration with lignocaine on postoperative pain after molar teeth extraction under general anesthesia. Anesthesia 1996; 51; 585-7.

161 - TSIRLIS A,T IAKOVIDIS DP, PARISSIS NA. Dry socket: frequency of occurrence after intraligamentary anesthesia. Quintessence Int 1992; 23: 575-7.

162 - TSUChIHASHI T, TAKatA Y, KUROKAWA H, MIURA K, MARUOKA Y, KAJ IYAMA M, FUJ ISHIMA M. Blood pressure response during dental surgery. Hypertension research, clinical and experimental 1996; 19: 189-94.

163 - Vahatalo K, Antila H, Lehtinen R. Articaine and lidocaine for maxillary infiltration anesthesia. Anesth Prog 1993; 40: 114-6.

164 - Vanderheyden PJ, Williams RA, Sims TN. Assessment of ST segment depression in patients with cardiac disease after local anesthesia. J Am Dent Assoc 1989; 119: 407-12.

165 - Viel E, Bruelle P, La Coussaye J E, Eledjam J J. Pharmacologie des Anesthésiques locaux. In Brasseur L. Douleurs, Maloine Editeur, Paris 1997

166 - Volpato MC, Ranali J , Amaral IMG, Demetrio CGB, ChALITA LVAS. Acute toxicity of lidocaine and prilocaine in combination with adrenaline and felypressin. Indian J Dent Tes 1999; 10: 138-44.

167 - Von TSAKIRIS A, BuHLmann A. Experimentelle Untersuchjungen beim Menschen über die wirkjung von vasopressin auf die Leberdurchblutung und den Portalen Druck. Hel Med Acta 1961; 4: 615-21.

168 - VReeland L, Reader A, Beck M, Meyers Weaver J. An evaluation of volumes and concentration of lidocaïne in human inferior alveolar nerve block. J Endod 1989; 15: 6-12.

169 - WAHL MJ. Local anesthetics and vasoconstrictors: myths and facts. Pract Period Aest Dent 1997; 9: 649-52.
170 - WAHL MJ . Demystifying medical complexities. Calif Dent Assoc J 2000; 28: 510-8.

171- WaLton RE, Garnick JJ. The periodontal ligament injection: histological effects on the periodontium in monkeys. J Endod 1982; 8: 22-6.

172 - WANG HH. The effects of 2-PHE, 8-Lys vasopressin, angiotensin, and nor-epinephrine on coronary blood flow and arterial blood pressure. Fed Proc 1964; 24 : 565.

173 - WATERS BD. Providing Dental treatment for patients with cardio-vascular disease. Ontario Dent 1995; 72: 24-32.

174 - Wong KC, Puerto AX, Puerto BA, Blatnick RA. Influence of imipramine and pargyline during halothane, enflurane or methoxyflurane anesthesia in dogs. Life Sci 1980; 27:2675-8.

175 - Wynn RL. Dental drug interactions with the greatest potential for serious adverse effects. Gen Dent 1994; 42: 116-7.

176 - WYNn RL. Dental considerations of patients taking appetite suppressants. Gen Dent 1997; 45: 324-8.

177 - WYNN RL. Hypertension medications and dental considerations. Gen Dent 2000; 48: 126-31.

178 - YAGUIELA J A. Local anaesthetics: a century of progress. Anesth Prog 1985; 32: 47-56.

179 - Yaguiela J A, Duffin SR, Hunt LM. DRUg INTERACTIONS and vasoconstrictors used in local anaesthetic solutions. Oral Surg Oral Med Oral Pathol 1985; 59: 565-71.

180 - YagUiela J A. Vasoconstrictors Agents for local anesthesia. Anesth Prog 1995; 42: 116-20.

181 - Yaguiela JA. Adverse Drug Interactions in dental practice. Interactions associated with vasoconstrictors. J Am Dent Assoc 1999; 130: 701-9.

182 - YAKSH TL, REDDY SV. Studies in the primate on the analgesic effects associated with intrathecal actions of opiates, alpha-adrenergic agonists and baclofen. Anesthesiology 1981; 54: 451-6.

183 - Yoshimura Y, OKa M, Kishimoto H, MatsuURA R, MISHIMA K. Hemodynamic changes during dental extraction and post-extraction bleeding in patients with prosthetic heart valves. Int J Oral Maxillofac Surg 1987; 16: 425-31.

184- Yuge M, KaWAmoto M, M ORIWAKI K. Balanced anesthesia today: the meanings of balanced anesthesia. The epidural anesthesia with general anesthesia. J J ap Soc Clin Anesth 1995; 153-5.

185 - Zhang C, Banting D, Gelb A, Hamilton J T. Effects of $\beta$ adrenoreceptors blockade with nadolol on the duration of local anesthesia. I Am Dent Assoc 1999; 130: 1773-80. 